\title{
Second virial coefficients of light nuclear clusters and their chemical freeze-out in nuclear collisions
}

\author{
K. A. Bugaev ${ }^{1,2}$ a, O. V. Vitiuk ${ }^{2,3}$, B. E. Grinyuk ${ }^{1}$, V. V. Sagun ${ }^{1,4}$, N. S. Yakovenko ${ }^{2}$, O. I. Ivanytskyi ${ }^{1,4}$, G. M. \\ Zinovjev $^{1}$, D. B. Blaschke ${ }^{5,6,7}$ E. G. Nikonov ${ }^{8}$, L. V. Bravina ${ }^{3}$, E. E. Zabrodin ${ }^{3,9}$, S. Kabana ${ }^{10}$, S. V. Kuleshov ${ }^{11}$, G. \\ R. Farrar $^{12}$, E. S. Zherebtsova ${ }^{7,13}$ and A. V. Taranenko ${ }^{7}$ \\ ${ }^{1}$ Bogolyubov Institute for Theoretical Physics, Metrologichna str. $14^{B}$, Kyiv 03680, Ukraine \\ ${ }^{2}$ Department of Physics, Taras Shevchenko National University of Kyiv, 03022 Kyiv, Ukraine \\ ${ }^{3}$ University of Oslo, POB 1048 Blindern, N-0316 Oslo, Norway \\ ${ }^{4}$ CFisUC, Department of Physics, University of Coimbra, 3004-516 Coimbra, Portugal \\ ${ }^{5}$ Institute of Theoretical Physics, University of Wroclaw, Max Born Pl. 9, 50-204 Wroclaw, Poland \\ ${ }^{6}$ Bogoliubov Laboratory of Theoretical Physics, JINR Dubna, Joliot-Curie Str. 6, 141980 Dubna, Russia \\ 7 National Research Nuclear University (MEPhI), Kashirskoe Shosse 31, 115409 Moscow, Russia \\ ${ }^{8}$ Laboratory for Information Technologies, Joint Institute for Nuclear Research, Dubna 141980, Russia \\ ${ }^{9}$ Skobeltsyn Institute of Nuclear Physics, Moscow State University, 119899 Moscow, Russia \\ ${ }^{10}$ Instituto de Alta Investigación, Universidad de Tarapacá, Casilla 7D, Arica, Chile \\ ${ }^{11}$ Departamento de Ciencias Físicas, Universidad Andres Bello, Sazié 2212, Piso 7, Santiago, Chile \\ 12 Department of Physics, New York University, New York, NY 10003, USA \\ ${ }^{13}$ Institute for Nuclear Research, Russian Academy of Science, 108840 Moscow, Russia
}

the date of receipt and acceptance should be inserted later

\begin{abstract}
Here we develop a new strategy to analyze the chemical freeze-out of light (anti)nuclei produced in high energy collisions of heavy atomic nuclei within an advanced version of the hadron resonance gas model. It is based on two different, but complementary approaches to model the hard-core repulsion between the light nuclei and hadrons. The first approach is based on an approximate treatment of the equivalent hard-core radius of a roomy nuclear cluster and pions, while the second approach is rigorously derived here using a self-consistent treatment of classical excluded volumes of light (anti)nuclei and hadrons. By construction, in a hadronic medium dominated by pions, both approaches should give the same results. Employing this strategy to the analysis of hadronic and light (anti)nuclei multiplicities measured by ALICE at $\sqrt{s_{N N}}=2.76 \mathrm{TeV}$ and by STAR at $\sqrt{s_{N N}}=200 \mathrm{GeV}$, we got rid of the existing ambiguity in the description of light (anti)nuclei data and determined the chemical freeze-out parameters of nuclei with high accuracy and confidence. At ALICE energy the nuclei are frozen prior to the hadrons at the temperature $T=175.1_{-3.9}^{+2.3} \mathrm{MeV}$, while at STAR energy there is a single freeze-out of hadrons and nuclei at the temperature $T=167.2 \pm 3.9 \mathrm{MeV}$. We argue that the found chemical freeze-out volumes of nuclei can be considered as the volumes of quark-gluon bags that produce the nuclei at the moment of hadronization.
\end{abstract}

PACS. 25.75.-q Relativistic heavy-ion collisions

05.70. Ce Thermodynamic functions and equations of state

64.30.-t Equations of state of specific substances

\section{Introduction}

The concept of hard-core repulsion plays an important role in the statistical mechanics of classical systems since, despite its simplicity, it allows one to correctly reproduce the basic properties of real gases at short distances. Its importance in describing the multiplicities of hadrons produced in the central high energy nuclear $(\mathrm{A}+\mathrm{A})$ collisions is beyond any doubts. In atomic physics it is clear that the hard core in the intermolecular interaction has its funda-

\footnotetext{
a e-mail: bugaev@th.physik.uni-frankfurt.de
}

mental origin in the Pauli exclusion principle and the electron exchange correlations between atoms and molecules (see, e.g., 1] and references therein) which allows one to predict the composition and thermodynamics of inertial fusion plasmas due to the account of the Pauli-blocking effect between atomic clusters [2]. However, the application of the fundamental Pauli principle on the quark level to account for a repulsive hard core in the interaction among hadrons is still in its infancy 3. The account of the Pauli blocking effect for light clusters in nuclear matter is meanwhile well-elaborated for not too high temperatures 
[4] and for applications to the composition of supernova matter [5,6], where usually excluded volume approaches are applied to account for light cluster abundances $[7,8,9]$. Within the quantum statistical approach, the second virial coefficient is addressed via a generalized Beth-Uhlenbeck equation of state which accounts for medium effects on the scattering phase shifts among clusters (cluster virial expansion [10]). The latter not only describes systematically the in-medium modification of the hard-core interaction, but ultimately leads to the Mott dissociation of the nuclear clusters due to Pauli blocking. The generalization of this successful quantum statistical approach to the higher temperatures by including all species of a hadron resonance gas and the treatment of repulsive Pauli-blocking effects on the basis of their fermionic quark substructure is a formidable task that has just been started [11. For the time being, one can already get interesting insights for the discussion of chemical freeze-out (CFO) of light clusters in the QCD phase diagram, in the context of ongoing discussions of the puzzle why these clusters freeze out in ultrarelativistic heavy-ion collisions at CERN and BNL according to predictions of the thermal statistical model at the same high temperature $T_{C F O} \approx 160 \mathrm{MeV}$ like all the other hadrons while their binding energies are at least an order of magnitude smaller.

This puzzle of the light nuclei production at LHC and RHIC has been discussed in many recent papers [12,13, 14, 15, 16, 17, 18, 19, 20, 21, 22, from both alternative points of view: the coalescence of nucleons (and hyperons, if applicable) in the final state after thermal freezeout of the hadrons on the one hand, and the CFO of the nuclei according to the thermal statistical model together with the other hadronic species directly in the vicinity of the hadronization transition in the QCD phase diagram on the other.

When drawing the lines for the Mott dissociation of light clusters as derived from the quantum statistical model into the QCD phase diagram one observes [23,24] that at the conditions of LHC and STAR experiments the medium modifications for nuclear clusters are not important, so that they can be expected to follow the ordinary thermal statistical model albeit including a hard core repulsion as in free space. Therefore, we devote the present work to extending the concept of hard core repulsion for hadronic systems with nuclear clusters in a thermodynamically consistent way and will apply it to the description of hadron and light cluster yields obtained in these experiments.

The real breakthrough in achieving a very high accuracy in the description of hadronic yields measured from the low AGS BNL collision energy $\left(\sqrt{s_{N N}}=2.7 \mathrm{GeV}\right)$ to the LHC CERN one $\left(\sqrt{s_{N N}}=2.76 \mathrm{TeV}\right)$ is related to the hadron resonance gas model (HRGM) with several hardcore radii of hadronic species [25, 26, 27, 28, i.e. with the multicomponent hard-core repulsion. Indeed, using just two extra parameters, the hard-core radii of pions $R_{\pi}$ and kaons $R_{K}$, in addition to the hard-core radii of baryons $R_{b}$ and the ones of other mesons $R_{m}$ which are traditionally employed in the HRGM, it was possible to achieve a very accurate description of all independent hadron multiplic- ity ratios measured prior to the LHC era with a $\chi^{2} / d o f$ which is in the range between 1.15 [26,27,28] and 0.96 [29]. The high accuracy achieved by the HRGM with multicomponent hard-core repulsion allowed us not only to elucidate the characteristics of the $\mathrm{CFO}$ of $\mathrm{A}+\mathrm{A}$ collisions, but also to reveal new irregularities of thermodynamic quantities at the $\mathrm{CFO}$ and to formulate new signals of two phase transitions [30, 31, 32, 33, 34] which are expected to exist in strongly interacting matter.

We have to remind that traditionally the CFO is defined as the moment after which the inelastic reactions stop to exist, while the evolution of hadronic matter is dominated by elastic reactions towards thermal freeze-out and decays of resonances 35.

However, the multicomponent versions of the HRGM based on the Van der Waals (VdW) approximation to the hard-core repulsion, i.e. which employ the classical second virial coefficients, are rather complicated and they take a lot of CPU time, since for $N$ different hard-core radii for each iteration of the fitting process of experimental data one has to solve the system of $N+1$ transcendental equations which involve hundreds of double integrals [25, 26, 27, 28, 29]. Therefore, the application of the multicomponent HRGM based on VdW approximation to cases of $N \gg 1$ is rather problematic. Fortunately, an entirely new and efficient approach to treat the multicomponent hard-core repulsion for large values of $N$ was invented in Ref. 36]. This novel approach based on the induced surface tension concept has two important advantages over the other multicomponent versions of the HRGM: first, the number of equations to be solved is always two and it does not depend on $N$ and, second, it allows one to go beyond the VdW approximation [37, 38, 39, 40. Note that the classical virial coefficients are traditionally denoted as the excluded volumes (per particle).

Despite the great achievements of the HRGM one important problem of the CFO was not resolved until recently. It is the CFO puzzle of light (anti)nuclei yields measured by the STAR RHIC collaboration in $\mathrm{Au}+\mathrm{Au}$ central collisions [42,43,44] at the center-of-mass collision energy $\sqrt{s_{N N}}=200 \mathrm{GeV}$ and the ones obtained recently by the ALICE CERN collaboration in $\mathrm{Pb}+\mathrm{Pb}$ collisions at the center-of-mass collision energy $\sqrt{s_{N N}}=2.76 \mathrm{TeV}$ 45, 46, 47.

There are many important aspects of the CFO puzzle of light (anti)nuclei yields [19,21, 22, 49, 50, 57, 58, 59, $60,61,62$ measured in $\mathrm{A}+\mathrm{A}$ collisions, but in our opinion the central one, is the value of their CFO temperature $T_{A}$. This is so, since without the reliable knowledge of their CFO temperature $T_{A}$ one cannot formulate a physically adequate model for the production of deuterons (d), helium-3 $\left({ }^{3} \mathrm{He}\right)$, helium-4 $\left({ }^{4} \mathrm{He}\right)$ and hyper-triton $\left({ }_{4}^{3} \mathrm{H}\right)$ and their antiparticles in $\mathrm{A}+\mathrm{A}$ collisions and a model of their thermalization as well. Other approaches in the literature which describe the production of nuclei in heavy ion collisions obtain estimates for $T_{A}$ using extensions of the HRGM that consider the nuclei as point-like particles [57] or assume the hard-core radius of all light (anti)nuclei to be equal to the hard-core radius of baryons $R_{b}$ [58. It is 
of interest to go a step further and aim to describe nuclei in a more realistic way.

In our previous work 60] a more elaborate HRGM has been presented that is based on the concept of induced surface tension [36, 37, 38. It uses an approximate expression for the hard-core radius of light (anti)nuclei denoted as bag model approximation (see below). This restriction was overcome recently in 62 , where it was shown that the bag model approximation can safely be used for piondominated matter. However, the derivation of the equation of state (EoS) which extends the induced surface tension concept to the classical second virial coefficients of light (anti)nuclei as suggested in [40] is a heuristic one.

In the present work we develop a mathematically rigorous treatment of a mixture of hadrons and light (anti)nuclei with hard-core repulsion based on the induced surface tension concept 36,37,38. In addition, with the help of this newly developed HRGM we analyze here not only the ALICE $\sqrt{s_{N N}}=2.76 \mathrm{TeV}$ data on light (anti)nuclei 45, 46. 47, but also the STAR $\sqrt{s_{N N}}=200 \mathrm{GeV}$ data [42,43 44. Our experience on achieving the accurate description of the hadronic data documented in Refs. [25, 26, 27, 28, 29, 30, 31, 33, 34, 37, 38 gives us a confidence that an essential improvement of the light nuclei data description will help the community to resolve the puzzles of the CFO of light nuclei.

The work is organized as follows. In Sect. 2 the mathematically rigorous derivation of the induced surface tension EoS for the mixture of hadrons and nuclei with classical second virial coefficients is presented. Sect. 3 contains the results on two models of the CFO of light (anti)nuclei produced in the central A+A collisions on LHC and RHIC. Sect. 4 is devoted to the discussion of the obtained results and summarizes our conclusions.

\section{Self-Consistent Treatment of Classical Excluded Volumes}

In this section, we briefly show how to extend the method of self-consistent treatment of classical systems with multicomponent hard-core interaction to the case of interaction of hadrons and light nuclei. It was introduced in 39 and successfully applied in [40, to mixtures of classical hard spheres and hard discs of different sizes.

There are three major reasons to consider the HRGM with multicomponent hard-core repulsion as the most realistic EoS of hadron matter at high temperatures and moderate particle number densities. First, a long time ago it was found that for temperatures below $170 \mathrm{MeV}$ and moderate baryonic charge densities (below the nuclear saturation density) the mixture of stable hadrons whose interaction is described by the quantum second virial coefficients behaves almost like a mixture of ideal gases of particles in which both the stable hadrons and their resonances are included, but the latter should have the averaged vacuum values of masses 48 . As it was demonstrated in Ref. 48 and recently discussed in Ref. 49, the main physical reason for this kind of behavior is rooted in an almost complete cancellation between the attractive and repulsive terms in the quantum second virial coefficients. Hence, the remaining deviation from the ideal gas (a weak repulsion) can be modeled by the classical hard-core repulsion.

Second, considering the HRGM as the EoS of hadronic matter one can be sure that its pressure will never exceed the one of the quark-gluon plasma. The latter may occur, if the hadronic phase is modelled as the mixture of ideal gases 38,51 . It is well-known that the number of spin-isospin degeneracies of all known hadrons and their resonances with the masses up to $2.6 \mathrm{GeV}$ is so large that, if one ignores the hard-core repulsion between them, at temperatures above $180 \mathrm{MeV}$ their pressure will be larger than the pressure of the quark-gluon plasma. An example of comparing the HRGM EoS with the lattice QCD results can be seen in Fig. 8 of Ref. 38.

Third, an additional and important reason to consider the HRGM as the hadronic matter EoS in the vicinity of CFO is a purely practical one: the hard-core repulsion is a contact interaction and, hence, the energy per particle of such an EoS coincides with the one of the ideal gas, even for the case of quantum statistics [52. Consequently, during the evolution of the system after CFO to the kinetic freeze-out one will not face a hard mathematical problem [53,54 to somehow "transform" the potential energy of interacting particles into their kinetic energy and into the masses of particles which appear due to resonance decays.

These are the main reasons which allow one to regard the HRGM as an extension of the statistical bootstrap model [55] supplemented by the hard-core repulsion, but for a truncated hadronic mass-volume spectrum, and which allow one to successfully apply it to the description of hadronic multiplicities measured in the central heavy ion collision experiments.

Although during last few years several valuable results were obtained with the help of HRGM [25, 26, 27, 28, 30, 31, $32,33,34,56$, at the moment the hard-core radii are well established for the most abundant hadrons, i.e. for pions, for the lightest $\mathrm{K}^{ \pm}$-mesons, for nucleons and for the lightest (anti) $\Lambda$-hyperons. Nevertheless, the HRGM based on classical virial coefficients is very successful in describing the properties of a hadron gas at CFO temperatures above $50 \mathrm{MeV}$, hence it is natural to apply it to the description of multiplicities of atomic nuclei measured in $\mathrm{A}+\mathrm{A}$ collision experiments instead of calculating their quantum virial coefficients.

However, even finding the classical excluded volumes of light (anti)nuclei consisting of $A$ baryons is, in general, a highly nontrivial task, since there is no well-developed formalism to calculate the cluster integrals of the particles which are clusters themselves. Due to this reason the usual Mayer procedure to calculate such cluster integrals cannot be used in the general case. Furthermore, even the classical excluded volumes of light (anti)nuclei with hadrons are known the next nontrivial task is to rigorously derive the corresponding system of equations which can be used for the actual fitting of the data. 


\begin{tabular}{|l|c|c|c|}
\hline Nucleus & $\begin{array}{c}\mathrm{R}_{\mathrm{rms}} \\
(\mathrm{fm})\end{array}$ & $\begin{array}{c}\text { classical distance } \\
L\left(R_{\mathrm{rms}}\right)\end{array}$ & $\begin{array}{c}\mathrm{L} \\
(\mathrm{fm})\end{array}$ \\
\hline deuteron & $2.1421 \pm 0.0088$ & $2 R_{\mathrm{rms}}$ & 4.280 \\
\hline triton & $1.7591 \pm 0.0363$ & $\sqrt{3} R_{\mathrm{rms}}$ & 3.047 \\
\hline${ }^{3} \mathrm{He}$ & $1.9661 \pm 0.0030$ & $\sqrt{3} R_{\mathrm{rms}}$ & 3.405 \\
\hline${ }^{4} \mathrm{He}$ & $1.6755 \pm 0.0028$ & $4 R_{\mathrm{rms}} / \sqrt{6}$ & 2.739 \\
\hline${ }_{\Lambda}^{3} \mathrm{H}$ & 4.9 (Ref. [65]) & $\sim \sqrt{3} R_{\mathrm{rms}}$ & 8.487 \\
\hline
\end{tabular}

Table 1. The rms radii of light nuclei $R_{\text {rms }}$ (2-nd column) taken from 64, except for the ${ }_{\Lambda}^{3} \mathrm{H}$ nuclei which is an estimate of Ref. 65. The 3-rd column shows the relation between the typical distance among the constituents $L$ and the rms radius $R_{\text {rms }}$ of light nuclei, whereas the 4-th column provides the actual estimates for $L\left(R_{\mathrm{rms}}\right)$. See text for details.

Fortunately, the light nuclei of $A$ baryons with $A \in$ $[2 ; 4]$ are roomy clusters, i.e. their root mean square (rms) radii $R_{\mathrm{rms}}=\sqrt{\left\langle r^{2}\right\rangle}$ are rather large 63,64 as one can see from the second column of Table 1 . This fact allows us to easily find out their classical second virial coefficient with the hadrons if the hard-core radii of all constituents of the considered nuclei are known. Assuming that the light nuclei of $A$ baryons can be considered as the quasi-classical particles which slowly move around the common centerof-mass on the distance $R_{\text {rms }}$, one can estimate the typical distance between the constituents $L\left(R_{\mathrm{rms}}\right)$ in such nuclei. For the (anti)deuteron the typical distance between the (anti)nucleons is $L\left(R_{\mathrm{rms}}\right) \simeq 2\left(R_{\mathrm{rms}}\right)$, while to estimate such a distance for triton, ${ }^{3} \mathrm{He},{ }_{\Lambda}^{3} \mathrm{H}$ and their antiparticles we suppose that they are the equilateral triangles. In this case, $R_{\text {rms }}$ is the radius of the circle described around the equilateral triangle and, hence, the classical distance between the constituents inside such nuclei is $L\left(R_{\mathrm{rms}}\right) \simeq \sqrt{3} R_{\mathrm{rms}} \simeq 1.732 R_{\mathrm{rms}}$. Similarly, for the ${ }^{4} \mathrm{He}$ nucleus and its antiparticle, we assume that the nucleons form an equilateral tetrahedron with the radius of the sphere described around it being $R_{\text {rms }}$. Then the classical distance between the constituents of the ${ }^{4} \mathrm{He}$ nucleus is $L\left(R_{\mathrm{rms}}\right) \simeq 4 R_{\mathrm{rms}} / \sqrt{6} \simeq 1.633 R_{\mathrm{rms}}$. These simple formulae and the actual estimates of $L\left(R_{\mathrm{rms}}\right)$ for different light nuclei are, respectively, given in the 3 -rd and 4 -th columns of Table 1.

Comparing the typical distances $L\left(R_{\mathrm{rms}}^{A}\right)$ between the constituents of $A$ baryons nuclei with the sum of largest hard-core diameter of hadrons $0.84 \mathrm{fm}$ [36, 37. and the

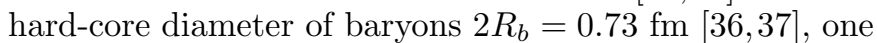
concludes that it is possible to freely translate the hadron with the hard-core radius $R_{h}$ around each of the nucleus constituent, i.e. a baryon of hard-core radius $R_{b}=0.365$ $\mathrm{fm}$, without touching any other constituent of this nucleus 62. Therefore, the classical second virial coefficient (excluded volume per particle) of a hadron and a nucleus of $A$ baryons can be written as

$$
b_{A h}=b_{h A}=A \frac{2}{3} \pi\left(R_{b}+R_{h}\right)^{3},
$$

where $R_{b}$ is the hard-core radius of baryons.
Similarly, we introduce the classical second virial coefficient (excluded volumes per particle) $b_{h_{1} h_{2}}$ of hadrons of radii $R_{h_{1}}$ and $R_{h_{2}}$ as

$$
b_{h_{1} h_{2}}=b_{h_{2} h_{1}} \equiv \frac{2}{3} \pi\left(R_{h_{1}}+R_{h_{2}}\right)^{3} .
$$

Now we consider a mixture of hadrons and light nuclei as Boltzmann particles with hard-core interaction. Neglecting for a moment the nucleus-nucleus interaction, i.e. assuming that $b_{A_{1} A_{2}}=0$, one can write the total excluded volume of such a mixture as

$$
V_{\text {excl }}^{\text {tot }}=\sum_{k \in h_{1}, A_{1}} \sum_{l \in h_{2}, A_{2}} N_{k} b_{k l} N_{l}
$$

where $N_{k}\left(N_{l}\right)$ is either the number of hadrons of sort $h$ or the number of nuclei of $A$ baryons. Note that in the sums in Eq. (3) the antiparticles are considered as the independent sorts of particles.

It is convenient to introduce the additional degeneracy of nuclei of $A$ baryons $g_{k A}$ and explicitly write the second virial coefficient (1) as

$$
\begin{aligned}
& \qquad \begin{aligned}
b_{k h_{l}} & =g_{k A} \frac{2}{3} \pi\left(R_{k}+R_{h_{l}}\right)^{3}=g_{k A} \frac{2}{3} \pi \times \\
& \times\left(R_{k}^{3}+3 R_{k}^{2} R_{h_{l}}+3 R_{k} R_{h_{l}}^{2}+R_{h_{l}}^{3}\right) \\
\text { where } \quad g_{k A} & \equiv A \delta_{k A}+\delta_{k h}, \text { and } g_{A A} R_{A}^{n}=A R_{b}^{n}
\end{aligned}
\end{aligned}
$$

where $\delta_{k A}$ and $\delta_{k h}$ are the Kronecker $\delta$ symbols.

Using the fact that the mean number of light nuclei $\left\langle N_{A}\right\rangle$ is very small compared to the mean number of all other hadrons $\sum_{h}\left\langle N_{h}\right\rangle$, i.e. $\left\langle N_{A}\right\rangle \ll \sum_{h}\left\langle N_{h}\right\rangle$, for light nuclei we can also write

$$
A\left\langle N_{A}\right\rangle \ll \sum_{h}\left\langle N_{h}\right\rangle
$$

which allows us to approximate Eq. (3) as

$$
\begin{aligned}
V_{\text {excl }}^{\text {tot }} & \simeq \frac{2}{3} \pi \sum_{k \in h_{1}, A_{1}} \sum_{l \in h_{2}, A_{2}} N_{k} g_{k A_{1}} \times \\
& \times\left(R_{k}^{3}+3 R_{k}^{2} R_{l}+3 R_{k} R_{l}^{2}+R_{l}^{3}\right) N_{l} g_{l A_{2}},
\end{aligned}
$$

where we substituted the binomial expression (4) for nucleus-hadron interaction and a similar binomial formula for the hadron-hadron interaction into Eq. (3). In addition in Eq. (7) the double summation is extended by adding the second degeneracy factor $g_{l A_{2}}$ to account for the nucleusnucleus interaction in a symmetric way which is convenient for further evaluation. Due to the inequality (6) which is valid for light nuclei the approximated Eq. (7) is rather accurate for $\mathrm{A}+\mathrm{A}$ collisions.

Combining the first term in the brackets of Eq. (7) with the last term, and the second term with the third one, it is possible to identically rewrite the total excluded volume 7 in a shorter form

$$
V_{\text {excl }}^{\text {tot }} \simeq \frac{4}{3} \pi \sum_{k \in h_{1}, A_{1}} \sum_{l \in h_{2}, A_{2}} N_{k} g_{k A_{1}}\left(R_{k}^{3}+3 R_{k}^{2} R_{l}\right) N_{l} g_{l A_{2}},
$$


which can be used to determine the mean excluded volume of the system per particle

$$
\begin{aligned}
& \bar{V}_{\text {excl }}=V_{\text {excl }}^{t o t} / \sum_{l \in h, A} N_{l} \simeq V_{\text {excl }}^{t o t} / \sum_{l \in h, A} N_{l} g_{l A} \simeq \\
& \simeq \sum_{k \in h_{1}, A_{1}} N_{k} g_{k A_{1}} V_{k}+\sum_{k \in h_{1}, A_{1}} N_{k} g_{k A_{1}} S_{k} \bar{R},
\end{aligned}
$$

where we introduced the eigen volume $V_{k}=\frac{4}{3} \pi R_{k}^{3}$ and eigen surface $S_{k}=4 \pi R_{k}^{2}$ of the particle of hard-core radius $R_{k}$ and the mean hard-core radius $\bar{R}$ defined as

$$
\bar{R}=\sum_{k \in h, A} N_{k} g_{k A} R_{k} / \sum_{l \in h, A} N_{l} .
$$

To obtain Eqs. (9)-(11) we, apparently, employed the inequality (6). In the thermodynamic limit Eqs. (10) and (11) enable us to self-consistently determine the EoS of the considered mixture within the VdW approximation.

To proceed further, we assume that for an infinite system one can replace all $N_{k}$ values in (11) by their statistical mean values $\left\langle N_{k}\right\rangle$ and write

$$
\bar{R} \rightarrow \sum_{k \in h, A}\left\langle N_{k}\right\rangle g_{k A} R_{k} / \sum_{l \in h, A}\left\langle N_{l}\right\rangle .
$$

where $\left\langle N_{l}\right\rangle$ will be calculated self-consistently using the grand canonical ensemble (GCE) partition function. This means that using $\bar{V}_{\text {excl }}(10$ with $\bar{R}$ defined by Eq. 12 one can calculate the GCE partition function regarding $R$ as a function of temperature $T$ and chemical potentials $\left\{\mu_{k}\right\}$ and afterwards one can find $\bar{R}$ from the calculated partition.

Denoting the chemical potential for the $k$-th sort of particles as $\mu_{k}$, one can write the GCE partition function as

$$
\begin{aligned}
& Z\left(T,\left\{\mu_{k}\right\}, V\right) \equiv \\
& \equiv \sum_{\left\{N_{k}\right\}}^{\infty}\left[\prod_{k \in h, A} \frac{\left[\phi_{k} e^{\frac{\mu_{k}}{T}}\left(V-\bar{V}_{e x c l}\right)\right]^{N_{k}}}{N_{k} !}\right] \theta\left(V-\bar{V}_{e x c l}\right)
\end{aligned}
$$

In Eq. 13 the thermal density $\phi_{k}$ of the $k$-th sort of particles contains the Breit-Wigner mass attenuation. In the Boltzmann approximation $\phi_{k}$ can be written as

$$
\begin{aligned}
\phi_{k}=g_{k} \gamma_{S}^{\left|s_{k}\right|} & \int_{M_{k}^{T h}}^{\infty} \frac{d m}{N_{k}\left(M_{k}^{T h}\right)} \frac{\Gamma_{k}}{\left(m-m_{k}\right)^{2}+\Gamma_{k}^{2} / 4} \times \\
& \times \int \frac{d^{3} p}{(2 \pi \hbar)^{3}} \exp \left[-\frac{\sqrt{p^{2}+m^{2}}}{T}\right],
\end{aligned}
$$

where $g_{k}$ denotes the degeneracy factor of the $k$-th sort of particle, $\gamma_{S}$ is its strangeness suppression factor [66, $\left|s_{k}\right|$ is the number of valence strange quarks and antiquarks in this sort of particle, while the factor

$$
N_{k}\left(M_{k}^{T h}\right) \equiv \int_{M_{k}^{T h}}^{\infty} \frac{d m \Gamma_{k}}{\left(m-m_{k}\right)^{2}+\Gamma_{k}^{2} / 4}
$$

denotes a normalization constant, in which $M_{k}^{T h}$ denotes the decay threshold mass of the $k$-th hadronic resonance, while $\Gamma_{k}$ denotes its width. Clearly, for the stable hadrons and light nuclei the width $\Gamma_{k}$ should be set to zero, which leads to the familiar expression for the thermal density

$$
\phi_{k}=g_{k} \gamma_{S}^{\left|s_{k}\right|} \int \frac{d p^{3}}{(2 \pi \hbar)^{3}} \exp \left[-\frac{\sqrt{p^{2}+m_{k}^{2}}}{T}\right] \text {. }
$$

We would like to stress that the Breit-Wigner ansatz for the mass attenuation is an approximation which is usually valid for relatively narrow resonances only. However, the expression for thermal density of unstable particles (14) in the spirit of a Beth-Uhlenbeck EoS [67] is valid for more general mass distributions which may replace this ansatz. For some dynamical models of hadron structure such as the NJL model, one could separate the resonant part of the interaction which would correspond to an unstable hadronic state and can be approximated by a BreitWigner ansatz and the residual, repulsive interaction 68 , 69,70. This is fortunate if the approach shall be combined with an excluded volume model for the short-range repulsion, in order to avoid a possible double counting. The generalized Beth-Uhlenbeck EoS can be rigorously derived for a mixture of hadron resonances 72,73 from a cluster decomposition of the Phi-functional approach [74, if the generalized Phi-functional belongs to the class of cluster two-loop diagrams [75, 11.

Note that the Heaviside step function $\theta$ in Eq. (13) is very important, since it ensures the absence of negative values of the available volume $\left(V-\bar{V}_{\text {excl }}\right)$ and provides the finite number of all particles for finite volume of the system $V$. However, due to its presence, the evaluation of the GCE partition function (13) is hard. To overcome this difficulty one should make the Laplace transformation with respect to $V$ to the isobaric partition (for an appropriate review see [76]) which is defined as

$$
\mathcal{Z}\left(T,\left\{\mu_{k}\right\}, \lambda\right) \equiv \int_{0}^{\infty} d V e^{-\lambda V} Z\left(T,\left\{\mu_{k}\right\}, V\right)
$$

Below we show that the isobaric partition $\mathcal{Z}\left(T,\left\{\mu_{k}\right\}, \lambda\right)$ can be found exactly by changing the integration variable $d V \rightarrow d\left(V-\bar{V}_{\text {excl }}\right)$. However, first of all it is necessary to define the quantities $\left\langle N_{k}\right\rangle$ in the GCE variables. In terms of the partial $\mu_{k}$-derivative of the partition $[13)$, one can define $\left\langle N_{k}\right\rangle$ as follows

$$
\left\langle N_{k}\right\rangle \equiv T \frac{\partial}{\partial \mu_{k}} \ln \left[Z\left(T,\left\{\mu_{l}\right\}, V\right)\right]
$$

In terms of definition (18) Eq. 12 for $\bar{R}$ can be cast as

$$
\bar{R}=\frac{\sum_{k \in h, A} g_{k A} R_{k} \frac{\partial}{\partial \mu_{k}} \ln \left[Z\left(T,\left\{\mu_{l}\right\}, V\right)\right]}{\sum_{k \in h, A} \frac{\partial}{\partial \mu_{k}} \ln \left[Z\left(T,\left\{\mu_{l}\right\}, V\right)\right]} .
$$


Changing the variable $d V \rightarrow d\left(V-\bar{V}_{\text {excl }}\right)$ in Eq. (17), one finds

$$
\begin{aligned}
& \mathcal{Z}\left(T,\left\{\mu_{k}\right\}, \lambda\right)=\int_{0}^{\infty} d V^{\prime} e^{-\lambda V^{\prime}} \times \\
& \times \sum_{\left\{N_{k}\right\}} \prod_{k \in h, A} \frac{1}{N_{k} !}\left[\phi_{k} e^{\frac{\mu_{k}}{T}} V^{\prime}\right]^{N_{k}} e^{-\lambda \bar{V}_{e x c l}} \theta\left(V^{\prime}\right) .
\end{aligned}
$$

Substituting into Eq. 20 the expression 10 for $\bar{V}_{\text {excl }}$, one gets

$$
\begin{aligned}
& \mathcal{Z}\left(T,\left\{\mu_{k}\right\}, \lambda\right)= \\
& =\int_{0}^{\infty} d V^{\prime} e^{-\lambda V^{\prime}} \sum_{\left\{N_{k}\right\}} \prod_{k \in h, A} \frac{\left[\phi_{k} e^{\frac{\mu_{k}}{T}-\lambda g_{k A}\left(V_{k}+\bar{R} S_{k}\right)} V^{\prime}\right]^{N_{k}}}{N_{k} !}= \\
& =\int_{0}^{\infty} d V^{\prime} \exp \left[V^{\prime}\left[\sum_{k \in h, A} \phi_{k} e^{\frac{\mu_{k}}{T}-\lambda g_{k A}\left(V_{k}+\bar{R} S_{k}\right)}-\lambda\right]\right] \cdot(21)
\end{aligned}
$$

Integration with respect to variable $d V^{\prime}$ in Eq. 21 can be done easily resulting in

$$
\mathcal{Z}\left(T,\left\{\mu_{k}\right\}, \lambda\right)=\frac{1}{\lambda-\mathcal{F}\left(\lambda, T,\left\{\mu_{k}\right\}\right)},
$$

where the function $\mathcal{F}\left(\lambda, T,\left\{\mu_{k}\right\}\right)$ which defines the system pressure in the thermodynamic limit is given by

$$
\mathcal{F}\left(\lambda, T,\left\{\mu_{k}\right\}\right)=\sum_{k \in h, A} \phi_{k} \exp \left[\frac{\mu_{k}}{T}-\lambda g_{k A}\left[V_{k}+S_{k} \bar{R}\right]\right] .
$$

The GCE partition function $(13)$ can be found now by the inverse Laplace transform

$$
\begin{aligned}
Z\left(T,\left\{\mu_{k}\right\}, V\right) & =\frac{1}{2 \pi i} \int_{\chi-i \infty}^{\chi+i \infty} d \lambda e^{\lambda V} \mathcal{Z}\left(T,\left\{\mu_{k}\right\}, \lambda\right)= \\
& =\left.\frac{e^{\lambda^{*} V}}{1-\frac{\partial \mathcal{F}}{\partial \lambda}\left(\lambda, T,\left\{\mu_{k}\right\}\right)}\right|_{\lambda=\lambda^{*}}
\end{aligned}
$$

As usual, in Eq. 24 the integration contour in the complex $\lambda$-plane is chosen to the right-hand side of the rightmost singularity $\lambda^{*}$, i.e. $\chi>\lambda^{*}$ (more details can be found in Ref. [76]). Since the number of hadronic states and light nuclei used in the HRGM is finite [36, 37, then the sum in Eq. (24) contains the finite number of terms and, hence, as shown in Ref. 76, the isobaric partition (24) has only the simple pole at $\lambda=\lambda^{*}$. The latter is a solution of the equation

$$
\lambda^{*}=\mathcal{F}\left(\lambda^{*}, T,\left\{\mu_{k}\right\}\right) .
$$

In the thermodynamic limit $V \rightarrow \infty$ from Eq. (24) one finds the system pressure as $p \equiv T \lambda^{*}$, since in this limit the GCE partition behaves as $Z\left(T,\left\{\mu_{k}\right\}, V \rightarrow \infty\right) \sim$ $\exp (p V / T)$ [77.
Using Eq. 25 one can write for the pressure

$$
p=T \sum_{k \in h, A} \phi_{k} \exp \left[\frac{\mu_{k}-p g_{k A}\left[V_{k}+\bar{R} S_{k}\right]}{T}\right],
$$

which should be supplemented by the equation for the mean hard-core radius $\bar{R}$. Using Eq. 24) one can rewrite Eq. 19 as follows

$$
\bar{R}=\frac{\sum_{k \in h, A} g_{k A} R_{k} \frac{\partial}{\partial \mu_{k}}\left[\lambda^{*} V-\ln \left(1-\frac{\partial \mathcal{F}}{\partial \lambda^{*}}\right)\right]}{\sum_{k \in h, A} \frac{\partial}{\partial \mu_{k}}\left[\lambda^{*} V-\ln \left(1-\frac{\partial \mathcal{F}}{\partial \lambda^{*}}\right)\right]} .
$$

In the thermodynamic limit $V \rightarrow \infty$ the terms $\ln \left(1-\frac{\partial \mathcal{F}}{\partial \lambda^{*}}\right)$ in Eq. 27) are small compared to the term $\lambda^{*} V$. Hence, finding the partial derivatives $\frac{\partial \lambda^{*}}{\partial \mu_{k}}$ from Eq. 25 , in the limit $V \rightarrow \infty$ one can rewrite Eq. (27) as

$$
\bar{R}=\frac{\sum_{k \in h, A} g_{k A} R_{k} \phi_{k} \exp \left[\frac{\mu_{k}-p g_{k A}\left[V_{k}+S_{k} \bar{R}\right]}{T}\right]}{\sum_{k \in h, A} \phi_{k} \exp \left[\frac{\mu_{k}-p g_{k A}\left[V_{k}+S_{k} \bar{R}\right]}{T}\right]} .
$$

With the help of equation 26 for pressure it is convenient to cast the last result in terms of the induced surface tension (IST) coefficient 36]

$$
\begin{aligned}
\Sigma & \equiv p \bar{R}= \\
& =T \sum_{k \in h, A} g_{k A} R_{k} \phi_{k} \exp \left[\frac{\mu_{k}-p g_{k A} V_{k}-\Sigma g_{k A} S_{k}}{T}\right]
\end{aligned}
$$

Rewriting equation for pressure similarly, one gets

$$
\begin{aligned}
p & =\sum_{k \in h, A} p_{k}= \\
& =T \sum_{k \in h, A} \phi_{k} \exp \left[\frac{\mu_{k}-p g_{k A} V_{k}-\Sigma g_{k A} S_{k}}{T}\right],
\end{aligned}
$$

where the partial pressures $\left\{p_{k}\right\}$ of each sort of particles are introduced for convenience.

The system of Eqs. (29) and (30) for the IST coefficient $\Sigma$ and pressure $p$, respectively, defines the EoS of the mixture of hadrons and light nuclei within the VdW approximation. Note that in contrast to the heuristic derivation of such a system suggested in 62 the present derivation of the system (29) and (30) is rigorous and well-controlled. The applicability range of the $\mathrm{VdW}$ approximation is, unfortunately, rather narrow and, therefore, its usage at the packing fractions $\eta=\sum_{k \in h, A} g_{k A} V_{k} \rho_{k}$ (here $\rho_{k}=\frac{\partial p}{\partial \mu_{k}}$ is the particle number density) above 0.12-0.15 may lead to problems with causality [37,38,78] (a typical example of acausal HRGM can be found in Ref. 79], see also its critique in Refs. 37, 38).

Fortunately, the applicability range of the system (29) and 30 can be extended to higher values of packing fractions in a simple way. The main idea of the IST approach 
[36, 37, 38, 39, 40 is that at high pressures the mean radius $\bar{R}$ in Eqs. $(26),(8)$ and 29 should be suppressed stronger than it is provided by the $\mathrm{VdW}$ approximation. Then for increasing pressure the mean radius $\bar{R}$ should gradually vanish leading to a reduction of the effective excluded volume of particle of $k$-th sort which is defined as

$$
\begin{aligned}
& V_{k}^{e f f}=g_{k A} \frac{\left(V_{k} p+S_{k} \Sigma\right)}{p} \rightarrow
\end{aligned}
$$

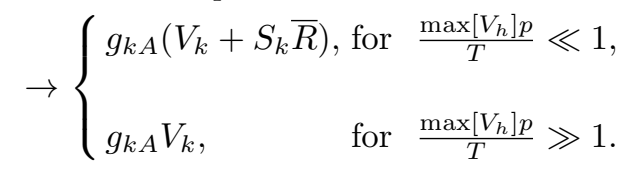

In other words, a gradual vanishing of the mean hard-core radius $\bar{R}$ should provide a slow transformation of the VdW (excluded volume) approximation, which is valid at low packing fractions $\eta \leq 0.1$, into the eigen volume approximation, which is valid at high packing fractions $\eta>0.5$ [77,80]. As suggested in Ref. [36] and verified in Refs. [37. 38, 39, 40 such an additional suppression of $\bar{R}$ can be obtained by replacing the term $\Sigma S_{k}$ on the right hand side of Eq. 29 as

$$
\Sigma S_{k} \rightarrow \Sigma S_{k} \alpha_{k}, \quad \text { where } \quad \alpha_{k}>1,
$$

where the auxiliary parameters $\alpha_{k}$ should be chosen in such a way that they describe the higher virial coefficients. Under this generalization Eq. (29) becomes

$$
\begin{aligned}
\Sigma & =\sum_{k \in h, A} \Sigma_{k}= \\
& =T \sum_{k \in h, A} g_{k A} R_{k} \phi_{k} \exp \left[\frac{\mu_{k}-g_{k A}\left(p V_{k}+\alpha_{k} \Sigma S_{k}\right)}{T}\right]
\end{aligned}
$$

where $\Sigma_{k}$ denotes the surface tension coefficient of $k$-th sort of particles. In this way one can account not only for the second virial coefficients, but also for the higher order virial coefficients as demonstrated for systems with singlecomponent hard-core repulsion in Refs. 37, 38, 39 and for two-component mixtures studied recently in 40 .

The reason to chose all the parameters $\alpha_{k}$ as $\alpha_{k}>1$ becomes apparent after analyzing the effective excluded volume (31). Indeed, substituting Eq. (33) into Eq. (31) one finds for hadrons

$$
V_{k}^{e f f} \equiv V_{k}+S_{k} \frac{\sum_{l \in h, A} p_{l} R_{l} e^{-\left(\alpha_{l}-1\right) S_{l} \Sigma / T}}{\sum_{n \in h, A} p_{n}} .
$$

This equation shows that in the limit of low packing fractions, i.e. for $\Sigma \max \left[S_{h}\right] / T \ll 1$, each exponential in Eq. 34 is $\exp \left[-\frac{\left(\alpha_{l}-1\right) S_{l} \Sigma}{T}\right] \simeq 1$ and, hence, one recovers the upper Eq. (31). However, it is easy to show that for high packing fractions an opposite inequality $\frac{\Sigma S_{k}}{T} \gg 1$ is valid for any $S_{k}>0$. In this case the condition $\alpha_{k}>1$ provides the vanishing of the mean radius $\bar{R} \equiv \frac{\Sigma}{p}$ and, hence, in this limit the effective excluded volume of each particle approaches its eigen volume, $V_{k}^{e f f} \rightarrow V_{k}$. Thus, at high packing fractions Eq. (34) leads to the lower Eq. (31).

The system (30), (33) is a generalization of the IST EoS derived in Ref. 40, for the classical hard spheres onto the multicomponent mixture of hard spheres (hadrons) and roomy classical clusters which are the light nuclei of $A$ baryons. As one can see from the derivation above such a generalization is not straightforward and contains some nontrivial steps. In particular, the inequality (6) played a crucial role in simplifying our derivation of the mean excluded volume per particle. Furthermore, the fact that the thermal density (14) of considered particles may, in principle, include the finite width opens an entirely new possibility to apply the present approach to the treatment of other roomy exotic clusters which have even larger width, than the hypertriton ${ }_{1}^{3} \mathrm{H}$ like, e.g., ${ }^{4} \mathrm{Li}$ and ${ }^{4} \mathrm{H}$ [6, 41].

In fact, the system (30), (33) can be generalized further in the spirit of Refs. [39, 40 in order to extend it to very high packing fractions $\eta \simeq 0.45-0.5$ by introducing into treatment the induced curvature tension.

In Refs. 37, 38 it is shown that even with a single parameter $\alpha_{k}=$ const $=\alpha=1.245$ Eqs. 30 , (33) for the classical hard spheres allows one to go beyond the VdW approximation, whereas in Ref. [40] one can find several examples on how two auxiliary parameters enables us to go far beyond the VdW approximation for two component classical systems. However, an extension of the system (30), (33) onto the quantum mechanical treatment of light (anti)nuclei in the spirit of Ref. 39] still remains a challenge for theoreticians.

\section{Analysis of light nuclei multiplicities measured in $A+A$ collisions}

The system (30), 33) is the IST EoS with classical excluded volumes of (light) nuclei and, hence, hereafter it is called IST EoS in order to distinguish it from another treatment of hard-core repulsion developed in 60,62. Although an approach of Refs. 60, 62 is approximative, nevertheless, we consider it as a complementary one to the IST model. It is based on the idea to introduce the equivalent hard-core radius $R_{A h}^{e q}$ of a pair $A h$ by equating the excluded volume $\frac{2}{3} \pi\left(R_{A h}^{e q}\right)^{3}$ with the equivalent hard-core radius $R_{A h}^{e q}$ to the actual excluded volume of such a pair $b_{A h}$ given by Eq. (1). Then we get the equivalent hard-core radius as 62 .

$$
R_{A h}^{e q}=A^{\frac{1}{3}}\left(R_{b}+R_{h}\right) .
$$

From the expression for $R_{A h}^{e q}$ one can determine the effective hard-core radius of a nucleus in a hadronic medium dominated by pions

$$
R_{A} \simeq R_{A \pi}^{e q}-R_{\pi} \simeq A^{\frac{1}{3}} R_{b}+\left(A^{\frac{1}{3}}-1\right) R_{\pi} \simeq A^{\frac{1}{3}} R_{b} .
$$

It is necessary to stress that this approximation is well justified for the $\mathrm{A}+\mathrm{A}$ of high energies by the fact that pions are the most abundant particles. In particular, this is the 
case for the LHC and the highest RHIC collision energies. The term $\left(A^{\frac{1}{3}}-1\right) R_{\pi}$ in Eq. 36 is a small correction to the effective hard-core radius of nuclei $R_{A} \simeq A^{\frac{1}{3}} R_{b}$, since the hard-core radius of pions $R_{\pi} \simeq 0.15 \mathrm{fm}$ [36, is essentially smaller than the one of baryons $R_{b}=0.365 \mathrm{fm}$ and the hard-core radii of kaons $R_{K}=0.395 \mathrm{fm}$ and other mesons $R_{m}=0.42 \mathrm{fm}$. Therefore, for low values of baryonic chemical potential (roughly for $\mu_{B}<T$ ) the pions are the least suppressed by the hard-core repulsion and, consequently, for any $A \leq 4$ the correction $\left(A^{\frac{1}{3}}-1\right) R_{\pi} \leq$ $0.088 \mathrm{fm}$ in Eq. (36) can be safely neglected for the piondominated hadronic medium.

The hard-core radius of light (anti)nuclei (36) is similar to the expression of the Bag Model radius (BMR) 81 of large bags of quark-gluon plasma and, hence, hereafter this model is called the BMR EoS. Despite the fact that it is an approximative approach, it is, however, simpler because with the help of the hard-core radius (36) the IST EoS allows one to treat the nuclei and hadrons exactly on the same footing. Moreover, a simultaneous use of IST and BMR approaches allows us to introduce a new strategy to locate the CFO of light (anti)nuclei. Since in the piondominated hadronic medium the BMR approach should give the same results as the IST, we have to search for the region of parameters at which both approaches provide a similar quality of the data description.

For the BMR approach the system (30), (33) should be slightly modified. Then formally considering the light (anti)nuclei as the primed sorts of hadrons $h_{A}^{\prime}$ (with $A=$ $2,3,4)$, we can write

$$
\begin{aligned}
p & =\sum_{k \in h, h_{A}^{\prime}} p_{k}=T \sum_{k \in h, h_{A}^{\prime}} \phi_{k} \exp \left[\frac{\mu_{k}-p V_{k}-\Sigma S_{k}}{T}\right] \\
\Sigma & =\sum_{k \in h, h_{A}^{\prime}} \Sigma_{k}= \\
& =T \sum_{k \in h, h_{A}^{\prime}} R_{k} \phi_{k} \exp \left[\frac{\mu_{k}-p V_{k}-\alpha_{k} \Sigma S_{k}}{T}\right]
\end{aligned}
$$

where the hard-core radii of primed hadrons are given by Eq. (36).

The partial values $p_{k}$ and $\Sigma_{k}$ entering the system (30), (33) (or (37), (38) ) allow one to write the particle number density of $k$-th sort of particle in a simple way

$$
\rho_{k} \equiv \frac{\partial p}{\partial \mu_{k}}=\frac{1}{T} \cdot \frac{p_{k} a_{22}-\Sigma_{k} a_{12}}{a_{11} a_{22}-a_{12} a_{21}} .
$$

For the system (37), (38) the coefficients $a_{k l}$ are given by 37 .

$$
\begin{aligned}
& a_{11}=1+\frac{4}{3} \pi \sum_{k \in h, h_{A}^{\prime}} R_{k}^{3} \frac{p_{k}}{T}, \quad a_{12}=4 \pi \sum_{k \in h, h_{A}^{\prime}} R_{k}^{2} \frac{p_{k}}{T}, \\
& a_{21}=\frac{4}{3} \pi \sum_{k \in h, h_{A}^{\prime}} R_{k}^{3} \frac{\Sigma_{k}}{T}, \quad a_{22}=1+4 \pi \sum_{k \in h, h_{A}^{\prime}} R_{k}^{2} \alpha_{k} \frac{\Sigma_{k}}{T},
\end{aligned}
$$

while in order to calculate the particle number densities for the system (30), (33) in Eqs. (40) and (41) one has to
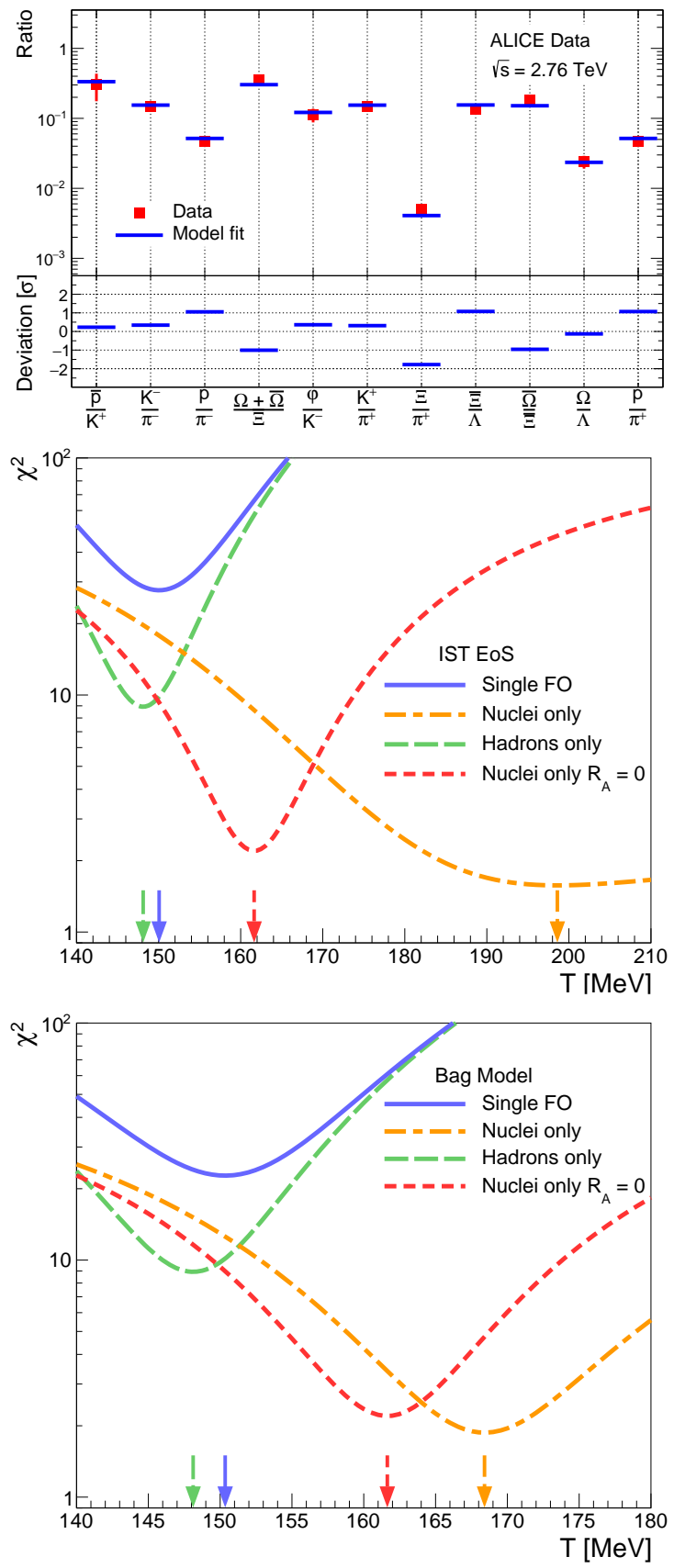

Fig. 1. Upper panel: Ratios of hadronic yields measured at $\sqrt{s_{N N}}=2.76 \mathrm{TeV}$ (symbols) vs. the results of IST EoS 30 , (33) (bars, for more details see the text). The CFO temperatures $T_{A}=T_{h}=150.1 \pm 1.9 \mathrm{MeV}$ are for the singe CFO IST EoS. Insertion shows the deviation of theory from data in the units of experimental error. Middle panel: Temperature dependence of $\chi_{\text {tot }}^{2}, \chi_{h}^{2}$ and $\chi_{A}^{2}$ for the IST EoS. Lower panel: Same as in the middle panel, but for the BMR EoS.

make the following replacements

$$
R_{h_{A}^{\prime}}^{2} \rightarrow A R_{b}^{2}, \quad R_{h_{A}^{\prime}}^{3} \rightarrow A R_{b}^{3},
$$

for the powers of hard-core radius of $A$ baryons nucleus. After finding all partial values $\left\{p_{k}\right\}$ and $\left\{\Sigma_{k}\right\}$, from the 
expressions $(39)-(41)$ one can determine the thermal yield $N_{k}^{t h}=V \rho_{k}$ of the $k$-th sort of particles. For hadrons, however, one has also to add the contribution coming from the decays of resonances. For the known branching ratios $B r_{l \rightarrow k}$ of hadronic decays $l \rightarrow k$ one can write the total yield of $k$-th sort of hadrons as follows

$$
N_{k}^{t o t}=V\left(\rho_{k}+\sum_{l \neq k} \rho_{l} B r_{l \rightarrow k}\right),
$$

where $V$ is the CFO volume. Since all details of the fitting process are well presented in the original works 37, 38, here we discuss the most important issues only.

To analyze the ALICE data [45,46, 47] we use the setup of Ref. 37, while for the analysis of the STAR data that of Ref. [38. The main difference in fitting the hadrons and the $A$-baryon nuclei is that for hadrons we use the ratios

$$
\mathcal{R}_{k l}^{\text {theo }}=\frac{\rho_{k}+\sum_{n \neq k} \rho_{n} B r_{n \rightarrow k}}{\rho_{l}+\sum_{n \neq k} \rho_{n} B r_{n \rightarrow l}},
$$

of yields of hadrons of sorts $k$ and $l$. On contrary for the $A$ baryon nuclei we employ the yields. Hence the total $\chi_{\text {tot }}^{2}(V)$ used in the present work is

$$
\begin{aligned}
& \chi_{\text {tot }}^{2}(V)=\chi_{h}^{2}+\chi_{A}^{2}(V)= \\
& =\sum_{k \neq l \in h}\left[\frac{\mathcal{R}_{k l}^{t h e o}-\mathcal{R}_{k l}^{\text {exp }}}{\delta \mathcal{R}_{k l}^{\text {exp }}}\right]^{2}+\sum_{A}\left[\frac{\rho_{A}(T) V-N_{A}^{\text {exp }}}{\delta N_{A}^{\text {exp }}}\right]^{2} .
\end{aligned}
$$

Here $\chi_{h}^{2}$ and $\chi_{A}^{2}$ denote, respectively, the mean deviation squared for hadrons and (anti)nuclei. Note that $\chi_{\text {tot }}^{2}(V)$ is a function of the CFO volume $V$. This is an important difference from our previous analyses of $25,26,27,29,37$, 38, which, as we will show below, allows us to elucidate the new details on the CFO of light (anti)nuclei. Since now on we also consider a single value $\alpha_{k}=1.25$ [37, 38 .

First, we apply the single CFO model to the ALICE data description. The hadronic data were taken from Refs. 82, 83, 84, 85. In the HRGM it is traditionally assumed that the CFO occurs for all particles simultaneously. The principal results are given in Table 2 and Figs. 1 and 2 To get these results we calculated the $\chi_{\text {tot }}^{2}$ using 2 fitting parameters, i.e. the CFO temperature and the CFO volume of nuclei $V=V_{A}$, for 11 hadronic ratios and 8 yields of light (anti)nuclei. All the chemical potentials are set to zero, while $\gamma_{s}=1$ is fixed according to Refs. 37, 38. The hard-core radii of hadrons are taken from our previous works [37,38] (are listed above). These values provide an excellent description of hadron yield ratios from AGS to LHC energies.

As one can see from Fig. 1 and from Table 2 the quality of ALICE data description obtained for the single CFO scenario is similar for the IST and BMR EoS. Moreover, the corresponding CFO temperatures are very similar, since the $\chi_{\text {tot }}^{2}$ is completely defined by the hadronic contribution to $\chi_{\text {tot }}^{2}$. Although the obtained overall description is satisfactory with $\chi_{\text {tot }}^{2} /\left.d o f\right|_{I S T} \simeq 1.627$ and $\chi_{\text {tot }}^{2} /\left.d o f\right|_{B M R} \simeq 1.336$, there are two surprising features in this scenario. First, $\chi_{\text {tot }}^{2} /\left.d o f\right|_{I S T}$ found by the advanced
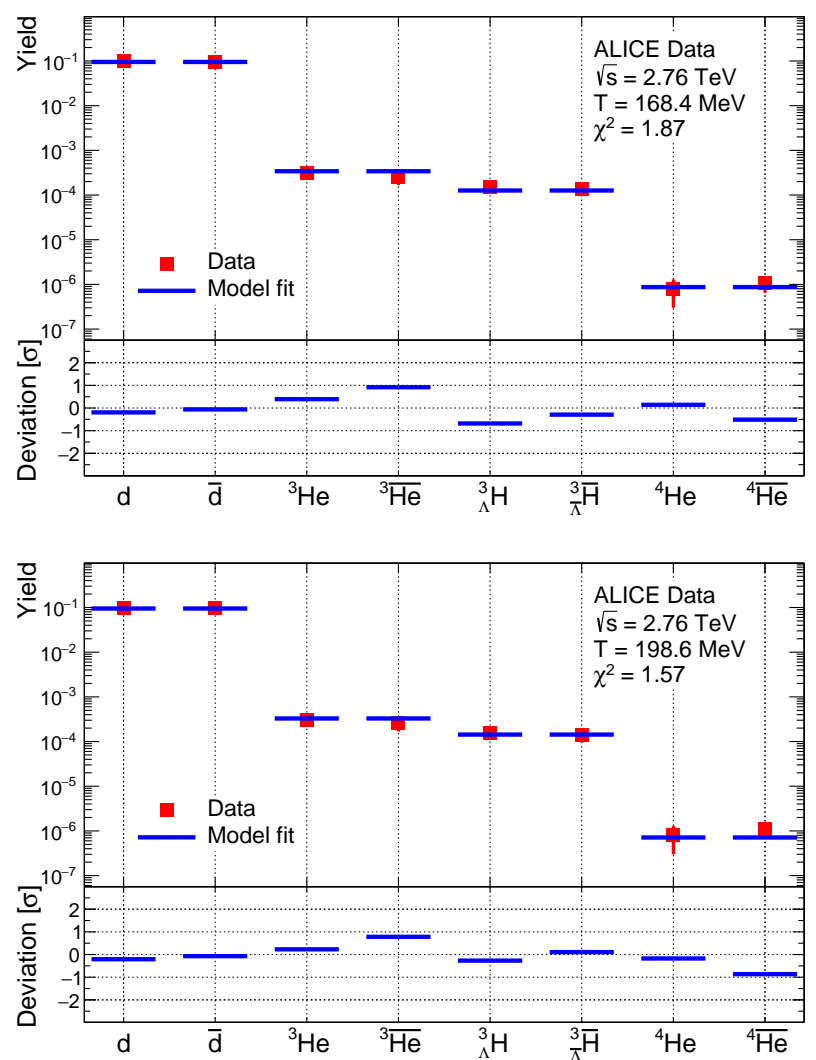

Fig. 2. The yields of nuclear clusters measured at $\sqrt{s_{N N}}=$ $2.76 \mathrm{TeV}$ vs. theoretical description in the scenario of separate CFO of light (anti)nuclei. Upper panel: The $\min \chi_{A}^{2}(V)$ corresponds to the BMR EoS (third row in Table 2). Lower panel: Same as in the upper panel, but for the IST EoS (forth row in Table 2).

model is somewhat larger than $\chi_{\text {tot }}^{2} /\left.d o f\right|_{B M R}$. Second, the CFO volumes of light (anti)nuclei are essentially larger than the CFO volume $V_{h}=\frac{N_{\pi^{+}}^{e x p}}{\rho_{\pi^{+}}+\sum_{l \neq k} \rho_{l} B r_{l \rightarrow \pi^{+}}} \simeq 8165 \pm$ $600 \mathrm{fm}^{3}$ of hadrons found from the experimental multiplicity of positive pions $N_{\pi^{+}}^{\text {exp }}$. This is best seen, when applying the new strategy to determine the common CFO volume for nuclei which is found to be $V_{A} \in[11100 ; 13260]$ $\mathrm{fm}^{3}$. Comparing $V_{A}$ with $V_{h}$ one finds that $\min V_{A} \simeq$ $11100 \mathrm{fm}^{3}$ is sizably larger than $\max V_{h} \simeq 8765 \mathrm{fm}^{3}$. This means that at the same CFO temperature the emission volume of hadrons and nuclei are rather different, i.e. the nuclei are freezing out in a much larger volume which means that there is no common hyper-surface of CFO. In our opinion, both of these features evidence for the internal inconsistency of the single CFO scenario at ALICE energy of collisions. Therefore, following the original idea of Ref. 60, we verify the hypothesis of separate CFO of light (anti)nuclei.

From Fig. 1 one can see that at high CFO temperatures the quantity $\chi_{A}^{2}\left(V_{A}\left(T_{A}\right)\right)$ has a deep minimum not only for the IST and BMR EoS, but even for the vanishing size of nuclei. In other words, the existence of a minimum of $\chi_{A}^{2}$ at high temperatures is a generic feature of the 


\begin{tabular}{lcccc}
\hline Description & $T_{h}, \mathrm{MeV}$ & $T_{A}, \mathrm{MeV}$ & $V_{A}, \mathrm{fm}^{3}$ & $\chi^{2} /$ dof \\
\hline Single CFO, BMR & $150.35 \pm 1.91$ & $150.35 \pm 1.91$ & $11241 \pm 2016$ & 1.336 \\
Single CFO, IST & $150.06 \pm 1.94$ & $150.06 \pm 1.94$ & $13357 \pm 2277$ & 1.627 \\
Separate CFO, BMR & $148.12 \pm 2.03$ & $168.41 \pm 5.60$ & $2997 \pm 1164$ & 0.675 \\
Separate CFO, IST & $148.12 \pm 2.03$ & $198.59 \pm 30.47$ & $1544 \pm 1027$ & 0.656 \\
\hline
\end{tabular}

Table 2. The results obtained by the advanced HRGM for the fit of ALICE data measured at $\sqrt{s}=2.76$ TeV. The CFO temperature of hadrons is $T_{h}$, the CFO temperature of light (anti)nuclei is $T_{A}$, while their CFO volume is $V_{A}$. The last column gives the fit quality.

advanced versions of HRGM. In the scenario of separate $\mathrm{CFO}$ of nuclei, there are three fitting parameters, namely the CFO temperatures of hadrons $T_{h}$ and nuclei $T_{A}$, and the CFO volume of nuclei $V_{A}$. As one can see from Table 2 and from Figs. 1 and 2 one can see that the hypothesis of separate CFO of nuclei provides an excellent fit with $\chi_{\text {tot }}^{2} /\left.d o f\right|_{I S T} \simeq 0.656$ and $\chi_{\text {tot }}^{2} /\left.d o f\right|_{B M R} \simeq 0.675$. Thus, compared to the single CFO scenario the value of $\chi_{\text {tot }}^{2} / d o f$ in this case decreased by $50 \%$.

However, the minimum of $\left.\chi_{A}^{2}\right|_{I S T}$ is located at essentially larger CFO temperature than the one found for the minimum of $\left.\chi_{A}^{2}\right|_{B M R}$. Moreover, the found $T_{A}$ for IST EoS is so large, that one can doubt the existence of hadrons and nuclei at this CFO temperature. Fortunately, with the help of the new strategy introduced in the preceding section one can resolve this problem easily. Indeed, similar results for the description of light (anti)nuclei by the IST and BMR EoS can be achieved in the vicinity of the common CFO temperature $T_{A}^{c o m}$ defined by the equality

$$
\begin{aligned}
& \left.\chi_{A}^{2}\left(V\left(T_{A}^{c o m}\right)\right)\right|_{I S T}=\left.\chi_{A}^{2}\left(V\left(T_{A}^{c o m}\right)\right)\right|_{B M R} \Rightarrow \\
& \Rightarrow T_{A}^{c o m}=175.1_{-3.9}^{+2.3} \mathrm{MeV},
\end{aligned}
$$

where the common CFO temperature still corresponds to a very accurate description of the ALICE data for light (anti)nuclei $\left.\chi_{A}^{2}\left(V\left(T_{A}^{c o m}\right)\right)\right|_{I S T} \simeq 3.2$ and $V_{A}^{\text {com }}=$ $V_{A}\left(T_{A}^{c o m}\right) \simeq 2660_{-1160}^{+1010} \mathrm{fm}^{3}$. The upper and lower deviations from $T_{A}^{c o m}=175.1 \mathrm{MeV}$ in Eq. (47) were found numerically by increasing the value of $\left.\chi_{A}^{2}\left(V\left(T_{A}^{c o m}\right)\right)\right|_{I S T} \simeq$ 3.2 on $1 \sigma$. Note that for $T_{A}^{c o m}=175.1 \mathrm{MeV}$ the total value of $\chi_{\text {tot }}^{2} /$ dof $=12.123 / 16 \simeq 0.758$ is rather small, i.e. it still corresponds to a highly accurate description of the ALICE data. Furthermore, the found range of $T_{A}^{c o m}$ is consistent with the values of CFO temperature found for the RHIC collision energy [37, 57, 56, 86] and it is a few $\mathrm{MeV}$ above the upper estimate for the cross-over temperature $T_{c o} \simeq 147-170 \mathrm{MeV}$ predicted by the lattice formulation of QCD at vanishing value of the baryonic chemical potential [87,88. Therefore, we are confident that the HRGM is applicable at these values of the common CFO temperature.

It is necessary to mention that the above numbers differ slightly from our preliminary results of a similar analysis reported in Ref. 62. The main difference is that in the present work we use the non-vanishing width for all hadronic resonances, while in Ref. 62 the solutions of systems (29), (30) and (37), (38) were found for zero width
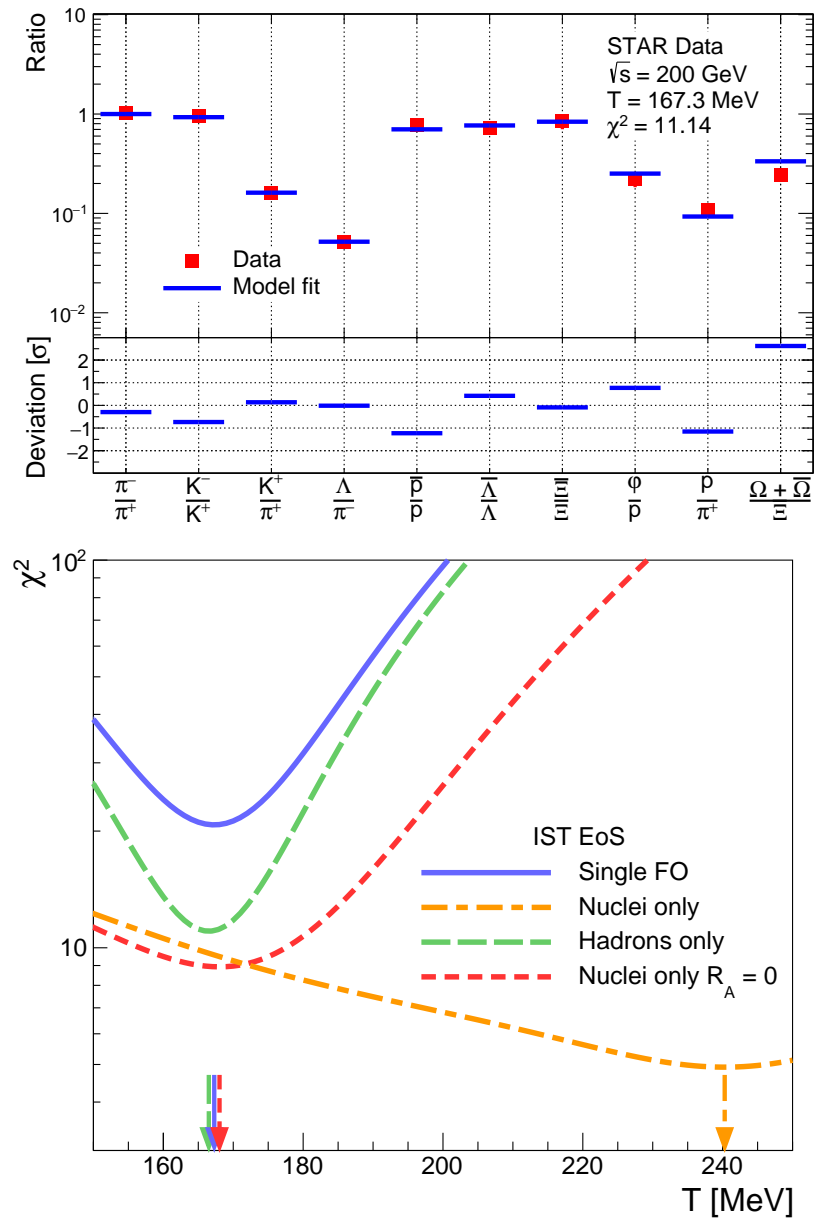

Fig. 3. Upper panel: Ratios of hadronic yields measured at $\sqrt{s_{N N}}=200 \mathrm{GeV}$ (symbols) vs. the results of IST EoS (bars) (30), (33) (for more details see the text). The CFO temperatures $T_{A}=T_{h}=167.28 \pm 3.93 \mathrm{MeV}$ are given for the singe CFO IST EoS. Insertion shows the deviation of theory from data in the units of experimental error. Lower panel: Temperature dependence of $\chi_{\text {tot }}^{2}, \chi_{h}^{2}$ and $\chi_{A}^{2}$ for the IST EoS.

of all hadronic resonances in order to fasten the fit process. However, the value of $T_{A}^{c o m}=175.1 \mathrm{MeV}$ found here and the result $T_{A}^{c o m}=174.6 \mathrm{MeV}$ found in 62 are practically the same, whereas its uncertainty determined here is a couple of $\mathrm{MeV}$ larger than in Ref. 62.

The results of a similar analysis of the STAR data measured at $\sqrt{s}=200 \mathrm{GeV}$ are presented in Table 3 and 
Figs. 3and 4. The STAR data consist of 10 hadronic ratios that are taken from Refs. 90,91,92 and are shown in the upper panel of Fig. 3 yields of (anti)deuterons 44, and 5 light (anti)nuclei yield ratios 42,43]. For the single CFO scenario, we have 3 fitting parameters, i.e. CFO temperature $T_{h}=T_{A}$, CFO baryonic chemical potential $\mu_{B}^{A}=\mu_{B}^{h}$ and the CFO volume of nuclei $V_{A}$. The strange chemical potential and the one of third projection of isospin are set to zero for simplicity, while $\gamma_{s}=1$ according to Ref. [38.

The results obtained for the hadronic ratios are depicted in the upper panel of Fig. 3, while the CFO temperature scan of $\left.\chi_{\text {tot }}^{2}\left(V\left(T_{A}=T_{h}\right)\right)\right|_{I S T}$ is shown in the lower panel of this figure. As one can see from Fig. 3 all hadronic ratios, except the ratio $\frac{\Omega+\bar{\Omega}}{\Xi}$, are well reproduced by the IST EoS. From Table 3 one can see that the CFO temperature $T_{h}$, the CFO baryonic chemical potential $\mu_{B}^{h}$ and $\chi_{\text {tot }}^{2} / d o f$ obtained for the IST and BMR EoS are practically the same. But the most striking result is that for the single CFO scenario, the value of common CFO volume $V_{A}^{\text {com }}=1898.5 \pm 157.5 \mathrm{fm}^{3}$ (see Table 3 ) is only 30 percent smaller compared to the corresponding value $V_{A}^{c o m} \simeq 2660_{-1160}^{+1010} \mathrm{fm}^{3}$ found above for the ALICE energy. We believe this is a remarkable finding, since the collision energy of the ALICE data is about 14 times larger than the one of the STAR data. At the same time for this scenario, the CFO volume of hadrons $V_{h}=2808 \pm 253 \mathrm{fm}^{3}$ found via the density of positive pions is slightly larger.

For the scenario of separate CFO of light (anti)nuclei, the CFO temperature of nuclei is substantially higher than the one of hadrons as one can see from Table 3. Although the higher value of $T_{A}$ provides a better description of the light (anti)nuclei yields as it is seen from Fig. 4. the value of $\chi_{\text {tot }}^{2}\left(V\left(T_{A}^{c o m}\right)\right) /\left.\operatorname{dof}\right|_{I S T}=\chi_{\text {tot }}^{2}\left(V\left(T_{A}^{c o m}\right)\right) /\left.d o f\right|_{B M R} \simeq$ 1.61 with $T_{A}^{\text {com }} \simeq 180 \pm 11.25 \mathrm{MeV}$ is about 10 percent larger than for the scenario of a single CFO. The reason is that for the scenario of separate CFO there are two additional parameters, namely the temperature of nuclei $T_{A}$ and their baryonic chemical potential $\mu_{B}^{A}$ were fitted in this case. As a result, the number of degrees of freedom in this case is $17-5=12$. Therefore, in contrast to the ALICE data, the STAR data do not demonstrate any preference for the separate CFO of light (anti)nuclei. Furthermore, in our opinion, there is no reason to expect that CFO of light (anti)nuclei can occur at the CFO temperatures above $175-180 \mathrm{MeV}$, since the hadronic description at those CFO temperatures is rather problematic according to the contemporary lattice version of QCD 87 88. The recent lattice QCD result for the continuumextrapolated chiral susceptibility at vanishing values of baryonic chemical potential define with high accuracy the pseudo-critical transition temperature $T_{p c}=156.5 \pm 1.5$ $\mathrm{MeV}$ [89] from its peak position. However, it also demonstrated that this peak has a full-width at half maximum of about $40 \mathrm{MeV}$. Therefore we conclude that the STAR data which favor the single CFO scenario with $\left.T_{A}^{c o m}\right|_{S T A R} \simeq$ $167.22 \mathrm{MeV}$ and $\left.V\left(T_{A}^{c o m}\right)\right|_{S T A R} \simeq 1898.5 \mathrm{fm}^{3}$ (see Table 3 ) is not in contradiction with the lattice QCD result of Ref. 89 .
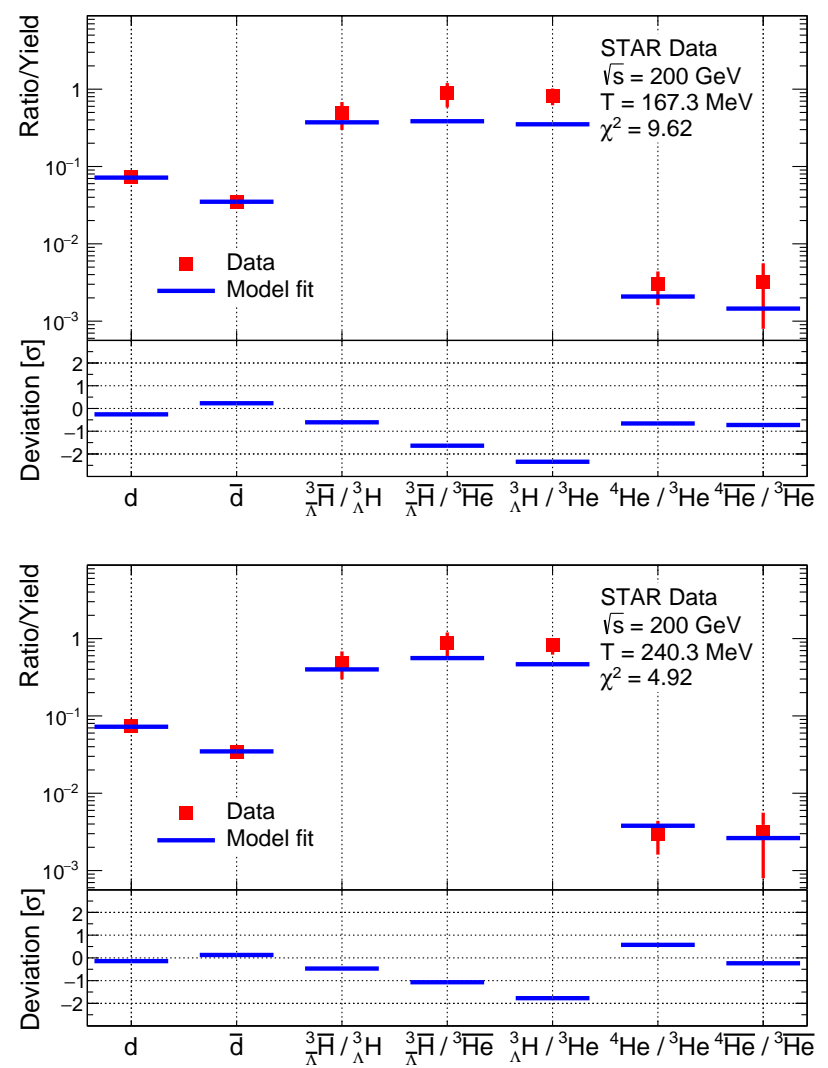

Fig. 4. Upper panel: Yield of (anti)deuteron and ratios of yields of light (anti)nuclei measured at $\sqrt{s_{N N}}=200 \mathrm{GeV}$ (symbols) vs. the results of IST EoS (bars) (30), (33) (for more details see the text). The temperatures $T_{A}=T_{h}=167.28 \pm$ $3.93 \mathrm{MeV}$ are for the singe CFO with IST EoS. Insertion shows the deviation of theory from data in the units of experimental error. Lower panel: Same as in the upper panel, but for the separate CFO with IST EoS for $T_{A}=240.29 \pm 21.38 \mathrm{MeV}$.

It is necessary to point out that the lower quality of the description of STAR data is generated by the ratios ${ }_{\Lambda}^{3} \overline{\mathrm{H}} /{ }^{3} \overline{\mathrm{He}}$ and ${ }_{\Lambda}^{3} \mathrm{H} /{ }^{3} \mathrm{He}$ (see Fig. 4). This is an old puzzle [93,94] which still awaits for its solution. It is clear, however, that an increase of the CFO temperature above $175-180 \mathrm{MeV}$ is not a viable solution and hence one has to look for another explanation.

The most intriguing question of this work is: how can one interpret the common CFO volume of light (anti)nuclei $V_{A}^{\text {com }}$ ? Of course, at the present stage of research, this question cannot answered with confidence, since neither the mechanism of light nuclei production nor the mechanism of their thermalization are well established. However, our educated guess is that the thermal production of light (anti)nuclei is most naturally caused by the hadronization of quark-gluon bags 60, 95. formed in A+A collisions which have the Hagedorn mass spectrum [55]. Since the Hagedorn mass spectrum is a perfect thermostat and a perfect particle reservoir [96] any particle or cluster emitted by the bags with such a mass spectrum will be produced in full chemical and thermal equilibrium with the emitting bag. Such a hypothesis is not only able, in prin- 


\begin{tabular}{|c|c|c|c|c|c|c|}
\hline Description & $T_{h}, \mathrm{MeV}$ & $T_{A}, \quad \mathrm{MeV}$ & $\mathrm{MeV}$ & $\mu_{B}^{A}, \mathrm{MeV}$ & $V_{A}, \quad \mathrm{fm}^{3}$ & $\chi^{2} / d o f$ \\
\hline Single CFO, BMR & $167.16 \pm 3.87$ & $167.16 \pm 3.87$ & $29.99 \pm 3.25$ & $29.99 \pm 3.25$ & $1692 \pm 364$ & 1.429 \\
\hline Single CFO, IST & $167.28 \pm 3.93$ & $167.28 \pm 3.93$ & $30.05 \pm 3.26$ & $30.05 \pm 3.26$ & $2155 \pm 411$ & 1.482 \\
\hline Separate CFO, BMR & $166.51 \pm 4.07$ & $178.62 \pm 14.63$ & $28.84 \pm 5.37$ & $32.63 \pm 4.94$ & $979 \pm 605$ & 1.607 \\
\hline Separate CFO, IST & $166.51 \pm 4.07$ & $240.29 \pm 21.38$ & $28.84 \pm 5.37$ & $44.08 \pm 6.81$ & $545 \pm 537$ & 1.330 \\
\hline
\end{tabular}

Table 3. The results obtained by the advanced HRGM for the fit of STAR data measured at $\sqrt{s}=200 \mathrm{GeV}$. The CFO temperature of hadrons (nuclei) is $T_{h}\left(T_{A}\right)$, the CFO baryonic chemical potential of hadrons (nuclei) is $\mu_{B}^{h}\left(\mu_{B}^{A}\right)$, while the CFO volume of nuclei is $V_{A}$. The last column gives the fit quality.

ciple, to explain the fact that the light nuclear clusters appear in full chemical and thermal equilibrium, but also the fact that the CFO temperatures extracted here from the ALICE and STAR data coincide. Moreover, in a recent preprint [97] based on a simplified transport model which, nevertheless, accurately takes into account the microscopic reactions between hadrons and heavy resonances with the Hagedorn mass spectrum it is shown that such an approach is able to reasonably well reproduce the ALICE data on hadronic and light nuclei multiplicities [45, 46, 47 with the common CFO temperature of hadrons and nuclei $T_{\text {com }}=149 \mathrm{MeV}$ for the Hagedorn temperature $T_{H}=167$ $\mathrm{MeV}$. Note that this value of $T_{\text {com }}$ almost coincides with the result $T_{h}=T_{A}=150.35 \pm 1.91 \mathrm{MeV}$ obtained within a single CFO scenario for the ALICE data (see Table 2). Unfortunately, the authors of Ref. 97 have not analyzed the case of different CFO temperatures for hadrons and light nuclei, but the results obtained in the present work clearly demonstrate that this scenario is more favorable.

Therefore, in accordance with our hypothesis, the found values of $V_{A}^{\text {com }}$ can be considered as the total volume of all quark-gluon bags from which the light (anti)nuclei are produced. If this is the case, we can predict that the entropy of quark-gluon bags produced at the collision energies $\sqrt{s_{N N}}=2.76 \mathrm{TeV}$ and $\sqrt{s_{N N}}=200 \mathrm{GeV}$ are related to each other as

$$
\frac{S_{A L I C E}}{S_{S T A R}} \simeq \frac{\left.V_{A}^{c o m} \cdot\left(T_{A}^{c o m}\right)^{3}\right|_{A L I C E}}{\left.V_{A}^{c o m} \cdot\left(T_{A}^{c o m}\right)^{3}\right|_{S T A R}} \simeq 1.609 .
$$

Since during the hydrodynamic expansion of a perfect fluid the entropy is approximately conserved, the ratio of entropies at $\mathrm{CFO}$ of nuclei should be equal to the ratio of initial entropies formed at the moment of thermalization of quark-gluon bags. Therefore, the relation between initial entropies 48 can be either verified by the hydrodynamic simulations or, alternatively, it can help to fix the value of initial energy density which is used in the integrated Hydro Kinetic Model 98.

In fact, the common CFO volumes obtained for two different energies of collision allow us to determine the number of emitting sources of nuclei. From the ratio of two common CFO volumes $\frac{\left.V\left(T_{A}^{c o m}\right)\right|_{A L I C E}}{\left.V\left(T_{A}^{c o m}\right)\right|_{S T A R}}=1.4015 \simeq \frac{7}{5}=\frac{14}{10}$ one can find the radius of emitting source $R_{A}^{\text {source }} \simeq 4.49$ $\mathrm{fm}$ for the number of 7 sources for the ALICE data and 5 sources for STAR data, or $R_{A}^{\text {source }} \simeq 3.566 \mathrm{fm}$ for the number of 14 sources for the ALICE data and 10 sources for the STAR data. Of course, it may be just a coincidence, but the radius of emitting source $R_{A}^{\text {source }} \simeq 3.566$ $\mathrm{fm}$ is just 0.25 percent smaller than the coalescence model parameter $\delta r=3.575 \mathrm{fm}$ used in Ref. 99] to model the formation process of light (anti)nuclei. Therefore, according to our hypothesis that the light (anti)nuclei are produced from the quark-gluon bags with Hagedorn mass spectrum at the moment of their hadronization the radius of the emitting source $R_{A}^{\text {source }} \simeq 3.566 \mathrm{fm}$ is, most likely, the radius of such bags. However, an additional verification of the found emitting source radius is necessary.

\section{Conclusions}

In this work, we suggested and exploited an entirely new strategy to elucidate the CFO parameters of light (anti)nuclei produced in $\mathrm{A}+\mathrm{A}$ collisions of high energy, in which the medium of secondary hadrons is dominated by pions. This strategy is based on two different approaches to model the hard-core repulsion between light nuclei and hadrons. The first approach is based on an approximate treatment of equivalent hard-core radius of roomy nuclear clusters and pions. The second approach is rigorously derived here using a self-consistent treatment of classical excluded volumes of light nuclei and hadrons. In other words, here we generalized the induced surface tension concept to the mixtures of hadrons of different hard-core radii and light (anti)nuclei of different sizes and masses, and derived the corresponding equation of state.

Since in the pion-dominated hadronic medium both approaches should give the same results by construction, we employed such a strategy to determine the simultaneous (common) description of the same experimental data by two different approaches. In all scenarios of CFO studied here we, indeed, always found the region where these two approaches provide a simultaneous and good description of the data. Such a strategy allows us to get rid of the existing ambiguity in the light (anti)nuclei data description and to determine the CFO parameters of nuclei in $\mathrm{A}+\mathrm{A}$ collisions of high energy with high confidence. In particular, for the ALICE data measured at $\sqrt{s_{N N}}=2.76$ $\mathrm{TeV}$ we found that the separate $\mathrm{CFO}$ of nuclei provides a very high accuracy in the description of hadronic multiplicity ratios and the light (anti)nuclei yields using only 3 fitting parameters with $\chi_{\text {tot }}^{2} / d o f \simeq 0.758$. The found CFO temperature of nuclei is $T_{A}^{c o m}=175.1_{-3.9}^{+2.3} \mathrm{MeV}$ and their 
CFO volume is $V_{A}^{\text {com }}=2660_{-1160}^{+1010} \mathrm{fm}^{3}$, while the CFO of hadrons occurs at essentially lower temperature.

On the contrary, from the analysis of the STAR data measured at $\sqrt{s_{N N}}=200 \mathrm{GeV}$, we found that the single CFO of hadrons and nuclei with 3 fitting parameters provides a better description which, in addition, is internally self-consistent. In this case the best description of the STAR data is achieved for the following CFO parameters of nuclei: $T_{A}^{c o m}=T_{h}=167.2 \pm 3.9 \mathrm{MeV}$, $V_{A}^{c o m}=1898.5 \pm 157.5 \mathrm{fm}^{3}$ and $\chi_{\text {tot }}^{2} /$ dof $\simeq 1.45$.

Based on the idea that the light (anti)nuclei are produced from the quark-gluon bags with an exponential mass spectrum, we interpret the found CFO volumes of nuclei as the sum of volumes of quark-gluon bags. From this interpretation, we estimated the ratio of initial entropy of thermalized bags for $\mathrm{A}+\mathrm{A}$ collision energies $\sqrt{s_{N N}}=200$ $\mathrm{GeV}$ and $\sqrt{s_{N N}}=2.76 \mathrm{TeV}$ and the number of the emitting sources of nuclei, which, in principle, can be verified by the hydrodynamic or hydro-kinetic approaches. Surprisingly, if the number of such sources is 14 for the ALICE energy and, consequently, 10 for the STAR energy, as it is required by their common $\mathrm{CFO}$ volumes, then the radius of emitting sources of nuclei is $3.566 \mathrm{fm}$, which practically coincides with the value of the coalescence distance used in a successful transport code simulating the production of nuclei 99 .

In the present work we demonstrate that the experimental data for the yields of hadrons and light nuclei produced in heavy-ion collisions at RHIC and LHC energies can be described with a very high accuracy, if one uses a formulation of the HRGM that employs the classical second virial coefficients corresponding to a hard-sphere model of nuclei and hadrons. At the same time it is shown that the determination of the CFO temperature of light nuclei is a rather delicate issue since the result depends on the underlying scenario of their CFO.

The simple model of hard spheres for the repulsive interactions between hadrons and nuclei as employed in the present work can of course only be considered as an intermediate step in our understanding of the formation of hadrons and light nuclei from a hadronizing quarkgluon plasma. Microphysical approaches should be further developed which treat hadrons and nuclei as multiquark clusters and would allow for a deeper understanding of the hadrochemistry on the quark level. One aspect of a description on this level would be the explanation of the short-range repulsion by quark Pauli blocking among hadrons (see, e.g., Ref. [3]), eventually augmented by repulsive multi-pomeron exchange forces that have proven essential to describe large-angle nucleus-nucleus scattering at the Fermi energy and in resolving the hyperon puzzle of neutron star structure 100 . From the further systematic analysis of light nuclei production measured in the heavy ion collision experiments a picture may emerge in which the puzzling result of a high CFO temperature finds its explanation by a hadronization of multi-quark states from the QGP, as it was already anticipated in Ref. 95. and emphasized again in Ref. [59] in the light of the recent experiments.

Author contributions. K.A.B. developed the idea behind this work and together with D.B.B. took the lead in writing the manuscript. O.V.V., B.E.G., V.V.S. and E.S.Z. performed fit of the experimental data on the light (anti)nuclei and hadrons. O.I.I., N.S.Y. and E.G.N. verified the analytical methods. Both N.S.Y. and S.V.K., helped in calculating the $\mathrm{CFO}$ volume of hadrons and designed the figures. G.M.Z., L.V.B., E.E.Z., S.K., G.R.F. and A.V.T. contributed to the interpretation of the results and provided a critical feedback. All authors discussed the results and contributed to the final manuscript.

Acknowledgments. The authors are thankful to Dmytro Oliinychenko for brining to our attention Ref. 44] and for illuminating discussions, and to Grigory Nigmatkulov and Ivan Yakimenko for the valuable comments. K.A.B. and G.M.Z. acknowledge support from the NAS of Ukraine by its priority project "Fundamental properties of the matter in the relativistic collisions of nuclei and in the early Universe" (No. 0120U100935). V.V.S. and O.I.I. are thankful for the support by the Fundação para a Ciência e Tecnologia (FCT), Portugal, by the project UID/04564/2020. The work of O.I.I. was supported by the project CENTRO01-0145-FEDER-000014 via the CENTRO 2020 program, and POCI-01-0145-FEDER-029912 with financial support from POCI, in its FEDER component and by the FCT/ MCTES budget via national funds (OE). The work of L.V.B. and E.E.Z. was supported by the Norwegian Research Council (NFR) under grant No. 255253/ F53 CERN Heavy Ion Theory, and by the RFBR grants 18-02-40085 and 18-02-40084. K.A.B., O.V.V., N.S.Ya. and L.V.B. thank the Norwegian Agency for International Cooperation and Quality Enhancement in Higher Education for the financial support under grants CPEA-LT-2016/10094 and UTF2016-long-term/10076. A.V.T. acknowledges partial support from RFBR under grant No. 18-02-40086 and from the Ministry of Science and Higher Education of the Russian Federation, Project "Fundamental properties of elementary particles and cosmology" No 0723-2020-0041. D.B.B. received funding from the RFBR under grant No. 18-02-40137. D.B.B. and A.V.T. acknowledge a partial support from the National Research Nuclear University "MEPhI" in the framework of the Russian Academic Excellence Project (contract no. 02.a03.21.0005, 27. 08.2013). The authors are grateful to the COST Action CA15213 "THOR" for supporting their networking.

\section{References}

1. W. Ebeling, D. Blaschke, R. Redmer, H. Reinholz and G. Röpke, J. Phys. A 42, 214033 (2009).

2. G. Röpke, D. Blaschke, T. Döppner, C. Lin, W. D. Kraeft, R. Redmer and H. Reinholz, Phys. Rev. E 99, no. 3, 033201 (2019).

3. D. Blaschke, H. Grigorian and G. Röpke, Particles 3, no.2, 477-499 (2020). 
4. S. Typel, G. Röpke, T. Klähn, D. Blaschke and H. H. Wolter, Phys. Rev. C 81, 015803 (2010).

5. M. Hempel, K. Hagel, J. Natowitz, G. Röpke and S. Typel, Phys. Rev. C 91, no. 4, 045805 (2015).

6. G. Röpke, Phys. Rev. C 101, no.6, 064310 (2020).

7. J. M. Lattimer and F. D. Swesty, Nucl. Phys. A 535, 331 (1991).

8. H. Shen, H. Toki, K. Oyamatsu and K. Sumiyoshi, Nucl. Phys. A 637, 435 (1998).

9. M. Hempel, J. Schaffner-Bielich, S. Typel and G. Röpke, Phys. Rev. C 84, 055804 (2011).

10. G. Röpke, N.-U. Bastian, D. Blaschke, T. Klähn, S. Typel and H. H. Wolter, Nucl. Phys. A 897, 70 (2013); arXiv:1209.0212 [nucl-th]].

11. N. U. F. Bastian, D. Blaschke, T. Fischer and G. Röpke, Universe 4, 67 (2018) and references therein.

12. S. Mrowczynski, Acta Phys. Polon. B 48, 707 (2017).

13. K. J. Sun, L. W. Chen, C. M. Ko and Z. Xu, Phys. Lett. B 774, 103-107 (2017).

14. K. J. Sun, L. W. Chen, C. M. Ko, J. Pu and Z. Xu, Phys. Lett. B 781, 499-504 (2018).

15. K. J. Sun, C. M. Ko and B. Dönigus, Phys. Lett. B 792 132-137 (2019).

16. V. Vovchenko, B. Dönigus and H. Stoecker, Phys. Lett. B 785, 171-174 (2018).

17. F. Bellini and A. P. Kalweit, Phys. Rev. C 99, no.5, 054905 (2019).

18. F. Bellini, K. Blum, A. P. Kalweit and M. Puccio, arXiv:2007.01750 [nucl-th]].

19. Y. Cai, T. D. Cohen, B. A. Gelman and Y. Yamauchi, Phys. Rev. C 100, no.2, 024911 (2019).

20. J. Aichelin, E. Bratkovskaya, A. Le Fèvre, V. Kireyeu, V. Kolesnikov, Y. Leifels, V. Voronyuk and G. Coci, Phys. Rev. C 101, no.4, 044905 (2020).

21. D. Oliinychenko, talk given at XXVIIIth Conference "Quark Matter 2019", arXiv:2003.05476v1 [hep-ph] and references therein.

22. S. Mrowczynski, arXiv:2004.07029v1 [nucl-th] and references therein.

23. D. Blaschke, A. V. Friesen, Y. B. Ivanov, Y. L. Kalinovsky, M. Kozhevnikova, S. Liebing, A. Radzhabov and G. Röpke, arXiv:2004.01159 [hep-ph].

24. D. Blaschke, G. Röpke, Y. Ivanov, M. Kozhevnikova and S. Liebing, Springer Proc. Phys. 250, 183 (2020).

25. D. R. Oliinychenko, K. A. Bugaev and A. S. Sorin, Ukr. J. Phys. 58, 211 (2013).

26. K. A. Bugaev, D. R. Oliinychenko, A. S. Sorin and G. M. Zinovjev, Eur. Phys. J. A 49, 30 (2013).

27. K. A. Bugaev et al., Europhys. Lett. 104, (2013) 22002.

28. K. A. Bugaev, A. I. Ivanytskyi, D. R. Oliinychenko, E. G. Nikonov, V. V. Sagun and G. M. Zinovjev, Ukr. J. Phys. 60, $181(2015)$

29. V. V. Sagun, Ukr. J. Phys. 59, 755 (2014).

30. K. A. Bugaev et al., Phys. Part. Nucl. Lett. 12, 238 (2015).

31. K. A. Bugaev et al., Eur. Phys. J. A 52, 175 (2016).

32. K. A. Bugaev et al., Eur. Phys. J. A 52, 227 (2016).

33. K. A. Bugaev et al., Phys. Part. Nucl. Lett. 15, 210 (2018).

34. K. A. Bugaev et al., EPJ Web of Conferences 204, 03001 (2019)

35. A. Andronic, P.Braun-Munzinger and J. Stachel, Nucl. Phys. A 772, 167 (2006) and references therein.

36. V. V. Sagun, A. I. Ivanytskyi, K. A. Bugaev and I. N. Mishustin, Nucl. Phys. A 924, 24 (2014).
37. V. V. Sagun et al., Eur. Phys. J. A 54, 100 (2018).

38. K. A. Bugaev et al., Nucl. Phys. A 970, 133 (2018).

39. K. A. Bugaev, Eur. Phys. J. A 55, 215 (2019).

40. N. S. Yakovenko, K. A. Bugaev, L.V. Bravina and E. E. Zabrodin, arXiv:1910.04889 [nucl-th] p. 1-13.

41. S. Bazak and S. Mrowczynski, Eur. Phys. J. A 56, no.7, 193 (2020).

42. STAR Collaboration (B. I. Abelev et al.), Science 328, No 5974, p. 58-62 (2010).

43. STAR Collaboration (H. Agakishiev et al.), Nature 473, No 7347 , p. $353-356$ (2011).

44. STAR Collaboration (J. Adam et al.), Phys. Rev. C 99, 064905 (2019).

45. ALICE Collaboration (J. Adam et al.), Phys. Rev. C 93 024917 (2016).

46. ALICE Collaboration (L. Ramonaet al.), AIP Conf. Proc. 1701, (1) 080009 (2016).

47. ALICE Collaboration (J. Adam et al.), Phys. Lett. B 754 360 (2016).

48. R. Venugopalan and M. Prakash, Nucl. Phys. A 546, 718 (1992).

49. E. Shuryak and J. M. Torres-Rincon, Phys. Rev. C 100 024903 (2019) and references therein.

50. E. Shuryak and J. M. Torres-Rincon, Phys. Rev. C 101 no.3, 034914 (2020).

51. L. M. Satarov, M. N. Dmitriev and I. N. Mishustin, Phys. Atom. Nucl. 72, 1390 (2009).

52. K. A. Bugaev, A. I. Ivanytskyi, V. V. Sagun, E. G. Nikonov and G. M. Zinovjev, Ukr. J. Phys. 63, 863 (2018) and references therein

53. K. A. Bugaev, Nucl. Phys. A 606, 559 (1996).

54. K. A. Bugaev, Phys. Rev. Lett. 90, 252301 (2003) and references therein

55. R. Hagedorn, Nuovo Cim. Suppl. 3, 147 (1965).

56. S. Chatterjee et al., Adv. High Energy Phys. 2015, 349013 (2015) and references therein.

57. J. Cleymans, S. Kabana, I. Kraus, H. Oeschler, K. Redlich and N. Sharma, Phys. Rev. C 84054916 (2011).

58. J. Stachel, A. Andronic, P. Braun-Munzinger and K. Redlich, J. Phys. Conf. Ser. 509, 012019 (2014).

59. A. Andronic, P. Braun-Munzinger, K. Redlich and J. Stachel, Nature 561, no.7723, 321-330 (2018).

60. K. A. Bugaev et al., J. of Phys. Conf. Series 1390, 012038 (2019).

61. P. Braun-Munzinger and B. Dönigus, Nucl. Phys. A 987 , 144 (2019) and references therein.

62. B. E. Grinyuk et al., arXiv:2004.05481v1 [hep-ph] (2020).

63. A. Bohr and B. Mottelson, Nuclear Structure, vol. 1 (Benjamin, New York, 1969).

64. I. Angeli and K. Marinova, At. Data Nucl. Data Tables 99, 69 (2013).

65. H. Nemura, Y. Suzuki, Y. Fujiwara, C. Nakamoto, Prog. Theor. Phys. 103, 929 (2000); arXiv:nucl-th/9912065

66. J. Rafelski, Phys. Lett. B 62, 333 (1991).

67. E. Beth and G. Uhlenbeck, Physica 4, 915 (1937).

68. J. Hüfner, S. P. Klevansky, P. Zhuang and H. Voss, Annals Phys. 234, 225 (1994).

69. A. Wergieluk, D. Blaschke, Y. L. Kalinovsky and A. Friesen, Phys. Part. Nucl. Lett. 10, 660 (2013).

70. D. Blaschke, M. Buballa, A. Dubinin, G. Röpke and D. Zablocki, Annals Phys. 348, 228 (2014).

71. D. Blaschke, A. Dubinin, A. Radzhabov and A. Wergieluk, Phys. Rev. D 96, no. 9, 094008 (2017). 
72. D. Blaschke, A. Dubinin and L. Turko, arXiv:1611.09845v2 [hep-ph].

73. D. Blaschke, A. Dubinin and L. Turko, Acta Phys. Polon. Supp. 10, 473 (2017).

74. G. Baym, Phys. Rev. 127, 1391 (1962).

75. B. Vanderheyden and G. Baym, J. Stat. Phys. 93, 843 (1998).

76. K. A. Bugaev and P. T. Reuter, Ukr. J. Phys. 52, 489 (2007) and references therein.

77. K. Huang, Statistical Mechanics (Wiley \& Sons, New York, 1967)

78. L. M. Satarov, K. A. Bugaev, I. N. Mishustin, Phys. Rev. C 91, 055203 (2015).

79. V. Vovchenko, H. Stöcker, J. Phys. G 44, 055103 (2017).

80. J. P. Hansen and I. R. McDonald, Theory of Simple Fluids (Academic Press, Amsterdam, 2006).

81. A. Chodos, R. L. Jaffe, K. Johnson, C. B. Thorn, V. F. Weisskopf, Phys. Rev. D 9, 3471 (1974).

82. B. Abelev et al. [ALICE Collaboration], Phys. Rev. C 88 (2013) 044910 .

83. B. B. Abelev et al. [ALICE Collaboration], Phys. Lett. B 728 (2014) 216; Erratum: [Phys. Lett. B 734 (2014) 409].

84. B. B. Abelev et al. [ALICE Collaboration], Phys. Rev. Lett. 111 (2013) 222301.

85. B. B. Abelev et al. [ALICE Collaboration], Phys. Rev. C 91 (2015) 024609

86. A. Andronic, P. Braun-Munzinger, K. Redlich and J. Stachel, J. Phys. Conf. Ser. 779, 012012 (2017).

87. Wuppertal-Budapest Collaboration (S. Borsanyi et al.), JHEP 1009, 073 (2010).

88. HotQCD Collaboration (A. Bazavov et al.), Phys. Rev. D 90, 094503 (2014).

89. A. Bazavov et al. [HotQCD], Phys. Lett. B 795 (2019), $15-21$.

90. J. Adams et al., Phys. Rev. Lett. 92, 112301 (2004).

91. J. Adams et al., Phys. Lett. B 612, 181 (2005).

92. A. Billmeier et al., J. Phys. G 30, S363 (2004).

93. A. Andronic, P. Braun-Munzinger, J. Stachel and $\mathrm{H}$. Stoecker, Phys. Lett. B 697, 203 (2011).

94. X. Xu and R. Rapp, Eur. Phys. J. A 55, 68 (2019); arXiv:1809.04024 $\mathrm{v} 2$ [nucl-th] and references therein.

95. G. F. Chapline and A. K. Kerman, MIT-CTP-695 (1978).

96. L. G. Moretto, K. A. Bugaev, J. B. Elliott and L. Phair, Europhys. Lett. 76, 402 (2006); LBNL preprint 56898.

97. K. Gallmeister and C. Greiner, arXiv:2007.08258 [hep$\mathrm{ph}]$.

98. V. Yu. Naboka, Iu. A. Karpenko and Yu. M. Sinyukov, Phys. Rev. C 93, 024902 (2016).

99. S. Sombun et al., Phys. Rev. C 99, 014901 (2019).

100. Y. Yamamoto, T. Furumoto, N. Yasutake and T. A. Rijken, Eur. Phys. J. A 52, no.2, 19 (2016). 